\title{
Creating large-scale city models from 3D-point clouds: a robust approach with hybrid representation
}

\author{
Florent Lafarge · Clément Mallet
}

the date of receipt and acceptance should be inserted later

\begin{abstract}
We present a novel and robust method for modeling cities from 3D-point data. Our algorithm provides a more complete description than existing approaches by reconstructing simultaneously buildings, trees and topologically complex grounds. A major contribution of our work is the original way of modeling buildings which guarantees a high generalization level while having semantized and compact representations. Geometric 3D-primitives such as planes, cylinders, spheres or cones describe regular roof sections, and are combined with mesh-patches that represent irregular roof components. The various urban components interact through a non-convex energy minimization problem in which they are propagated under arrangement constraints over a planimetric map. Our approach is experimentally validated on complex buildings and large urban scenes of millions of points, and is compared to state-of-the-art methods.
\end{abstract}

Keywords 3D-modeling - shape representation . urban scenes · point data · energy minimization . Markov Random Field

\section{Introduction}

\subsection{Problem statement}

The 3D-modeling of urban scenes is a topic of major interest in computer vision. Driven by new virtual applications, this research domain has considerably pro-

Florent Lafarge

INRIA, Sophia Antipolis, France

E-mail: Florent.Lafarge@inria.fr

Clément Mallet

Université Paris Est, Saint Mandé, France

E-mail: Clement.Mallet@ign.fr gressed during the last decade as underlined in recent studies (Zhu and Kanade, 2008; Mayer, 2008; Haala and Kada, 2010).

A part of the existing methods is devoted to street level modeling from ground or oblique-view data. These works propose accurate facade 3D-models which are particularly useful for the ground based navigation systems. They can be obtained from various types of data such as multi-view images (Dick et al., 2004; Strecha et al., 2008; Furukawa et al., 2009; Sinha et al., 2009; Vu et al., 2009; Vanegas et al., 2010), laser scans (Banno et al., 2008; Chen and Chen, 2008; Frueh and Zakhor, 2004) or video (Pollefeys et al., 2008; Gallup et al., 2010). Recently, several works have addressed the challenging problem of scene reconstruction from internet photo collections (Agarwal et al., 2009; Frahm et al., 2010; Furukawa et al., 2010). They generate impressive clouds of 3D-points which, nevertheless, contain outliers and have spatial distributions highly heterogeneous. Other works propose large city descriptions and offer complementary advantages to the street level representations, in particular fine roof descriptions. Such city descriptions are usually obtained either from airborne data for reconstructing in 3D existing landscapes, or from urban grammars in order to artificially create realistic cities as, for example, the procedural model proposed by Muller et al. (2006). These works are crucial for a large range of applications, from virtual globe visits to urban planning through to video games. We focus here on large-scale city modeling problems from aerial data, in particular from point set data generated by airborne geo-located acquisition systems. Note that, for such problems, the scene scale is supposed to be known as input point clouds are generated in the metric system. 


\subsection{Related works from point set data}

Most of the existing city modeling approaches directly or indirectly tackle the problem through point cloud analysis.

Digital Surface Models (DSM), which are 2.5D representations depending on a Z-direction, constitute structured point clouds having a regular point distribution in the XY-plane well adapted to aerial-based city modeling. Zebedin et al. (2008) and Lafarge et al. (2010a) generate DSMs from Multi-View Stereo (MVS) imagery in order to model buildings by polyhedral structures. The latter use a Constructive Solid Geometry (CSG) based approach by reconstructing a building as an assembling of parametric 3D-blocks, the former propose to partition a building in small 2D-polygons which are then labeled by graph-cut optimization.

Other approaches consider unstructured point clouds directly generated from laser/Lidar systems (Vosselman et al., 2005; Tse et al., 2007; Matei et al., 2008; Poullis and You, 2009; Toshev et al., 2010; Zhou and Neumann, 2010) or MVS imagery (Chauve et al., 2010). Such data have spatially heterogeneous point distributions without induced neighborhood relationships between the points, and contain outliers, especially when generated from MVS imagery. Vosselman et al. (2005) present a semi-automatic approach using an interactive segmentation of the parcel boundaries on which are fitted flat, gable, or hip roofs. Matei et al. (2008) and Poullis and You (2009) propose flat roof models adapted to Manhattan World environments (Coughlan and Yuille, 2000). Both approaches focus on segmenting the buildings and simplifying their boundaries, either by estimating building orientations (Matei et al., 2008) or by using statistical considerations (Poullis and You, 2009). Tse et al. (2007) identify some building components from a Delaunay triangulation of the point data which are then combined to model simple roof structures. A more general building representation is proposed by Zhou and Neumann (2010) who use a mesh simplification procedure based on dual contouring. Although this approach wins in terms of generalization, semantic information is lost: a simple planar roof section can be described by many mesh facets with different normal orientations.

\subsection{Motivations}

These approaches provide convincing 3D-models but have some important limitations. Firstly, strong urban prior on orthogonality, symmetry and roof typology are frequently introduced to reduce the solution space, and thus the problem complexity. These assumptions are usually efficient for Manhattan World environments but become penalizing for less well-organized urban landscapes having high variations of roof structures such as the areas presented in Section 6. Secondly, these methods provide a sparse description of urban scenes. They are focused on the building modeling task and disregard all the other objects which can be found in an urban scene such as trees, or even sometimes ground surfaces by assuming a constant altitude over the global scene. Thirdly, these models are each designed for a specific type of input data, and the resulting quality generally falls down when modifying data specifications. For instance, the mesh simplification algorithm proposed by Zhou and Neumann (2010) is of limited interest with point clouds of low densities, as well as the CSG-based approach of Lafarge et al. (2010a) with unstructured point sets generated from laser or MVS.

\subsection{Contributions}

We propose an algorithm which brings solutions to address the problems mentioned above. Our method presents several significant contributions to the field.

- More complete models of unspecified urban environments: we do not simply reconstruct the buildings: a more complete representation is provided by modeling vegetation and topologically complex grounds. Moreover, our method is adapted to various types of urban landscapes, from financial districts of big cities to small mountainous villages, including historical towns with old buildings of architectural interest. Besides, it is robust on a large range of point data having different point densities and various sensor characteristics.

- Hybrid reconstruction of buildings: the modeling of the buildings combines geometric 3D-primitives such as planes, cylinders, spheres or cones to represent standard roof sections and mesh-patches to describe more irregular roof components. Thus, 3D-models provide urban details while being semantized and compact. These two different types of 3D-representation tools interact through a non-convex energy minimization problem. This idea has been originally proposed in former works (Lafarge et al., 2010b) in order to reconstruct facades from MVS images and has revealed a high potential.

- 2.5D-arrangement scheme for the urban structures: a general formulation for the roof section arrangement problems is presented, the first to date to our knowledge which works in non-restricted contexts, i.e. with (i) unspecified primitives, (ii) various types of urban objects interacting in the scene, and (iii) unknown build- 


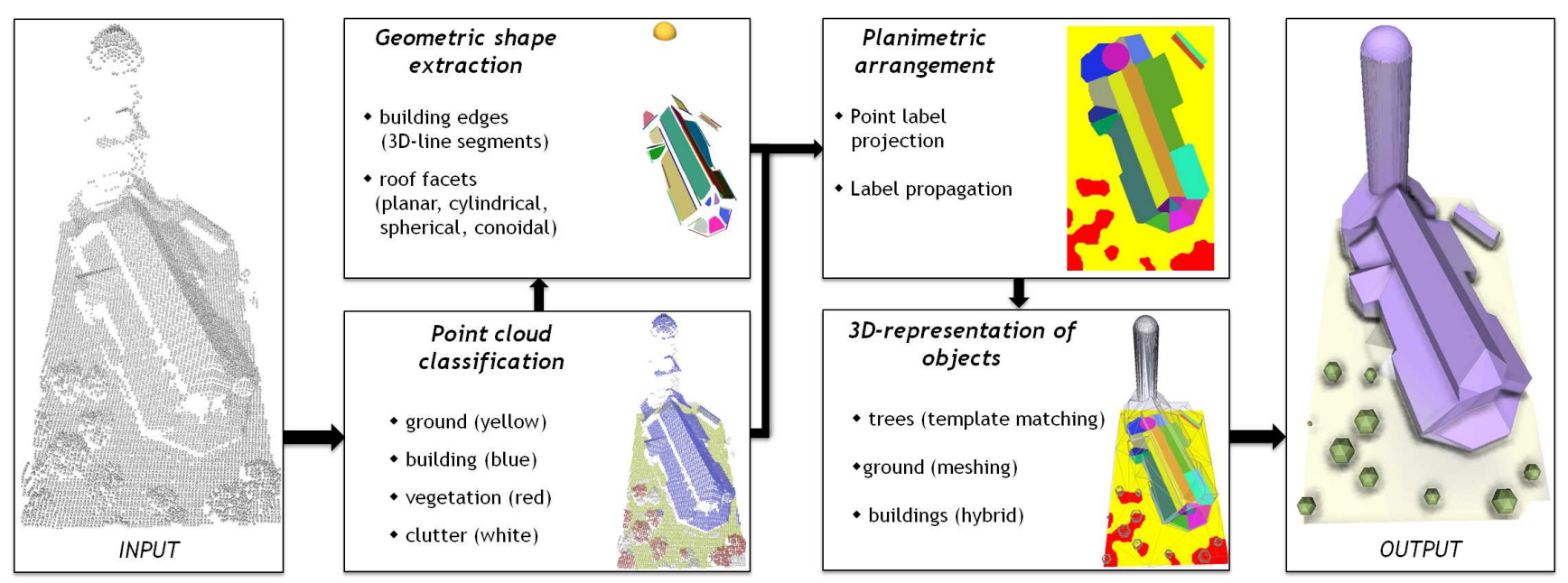

Fig. 1 Overview of the proposed approach - Our algorithm digests large amounts of 3D-points in order to provide a compact and semantized representation of urban environments including atypical buildings, trees, and topologically complex grounds.

ing contours. This 2.5D-arrangement scheme allows the combination of parametric 3D-shapes as well as unspecified urban components in a planimetric label map while imposing structural constraints.

A four-step strategy, illustrated in Fig. 1, is adopted. First, the point cloud is classified using an unsupervised method presented in Section 2. Four classes are distinguished: ground, building, vegetation and clutter. The second step, detailed in Section 3, consists in extracting geometric primitives such as 3D-line segments, planes or cylinders from the point set classified as building by a fast process. Section 4 constitutes the key element of the system in which the geometric primitives and the other urban components are arranged in a common planimetric map through a multi-label energy minimization formulation. In the last stage, the various urban objects are represented in 3D using template fitting and meshing procedures explained in Section 5. Experimental results on complex urban structures and various types of large urban scenes are presented and commented in Section 6, as well as a comparison from laser-based and MVS-based input data. This paper extends the work presented in (Lafarge and Mallet, 2011) by detailing the different steps of the method and its implementation, by presenting new results and comparisons as well as analyzing the impact of parameters and input types on the result quality.

\section{Point cloud classification}

Four classes of interest are defined: building, vegetation, ground and clutter. The class vegetation represents the trees which have a non negligible size at the city scale, i.e. with a height of several meters, excluding the shrubs. The class clutter corresponds to the outliers contained in the data, to small urban components which temporarily perturb the scene (e.g. cars, fences, wires, roof antennas, cranes), and to the vertical structures such as facades because these have a sparse and irregular point repartition penalizing the scene understanding. This class also includes water corresponding to river networks for which the point distribution is very sparse. Note that, in the case of urban scenes containing non-negligible sea areas, a fifth class is required to efficiently extract water since the point distribution is denser and needs to be discriminated by additional attributes (Carlberg et al., 2009).

A neighboring relationship is defined to create spatial dependencies between the 3D-points. Two points are neighbors if their Euclidean distance is inferior to a certain value, in practice $2 \mathrm{~m}$ (spherical neighborhood).

\subsection{Discriminative features}

For each point, several geometric attributes are computed in order to distinguish the four classes of interest.

- Local non-planarity $f_{p}$ represents the quadratic distance between the point and the optimal 3D-plane computed among its neighbors. Low values typically correspond to ground and building roofs.

- Elevation $f_{e}$ allows the distinction between the ground and the other classes. This feature corresponds to the height difference between the point and its planimetric projection on an elevation map of the ground estimated by a standard algorithm (Briese et al., 2002). 

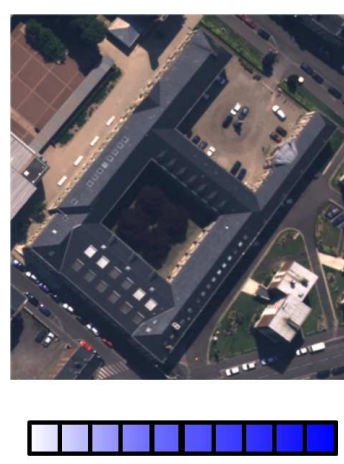

0

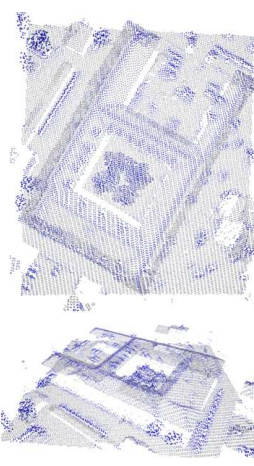

non-planarity $f_{p}$

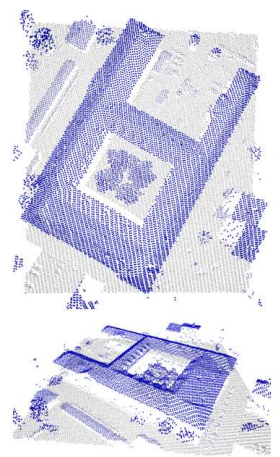

elevation $f_{e}$

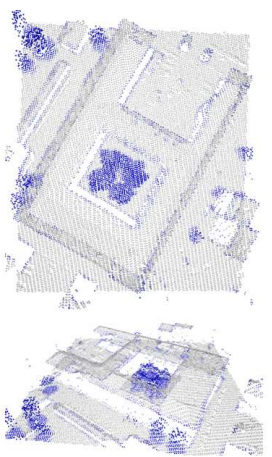

scatter $f_{s}$

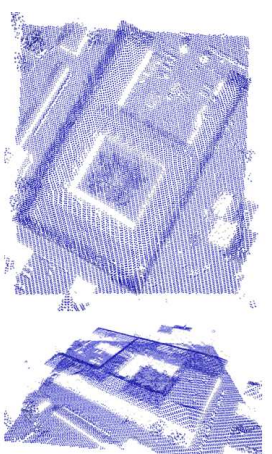

grouping $f_{g}$

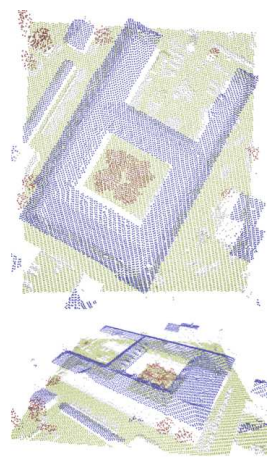

classification

Fig. 2 Behavior of the discriminative features- (from left to right) an aerial picture of the scene (not used) containing a building and its surrounding area, input clouds with the points colored according to the response of the features [color code: white=low response, blue $=$ high response], and the classification result [color code: blue=building, red=vegetation, yellow $=$ ground and white $=$ clutter . Each feature brings a specific type of information such that the combinations of the features allow the distinction of the different classes in the input point cloud. In particular, note the differences between $f_{s}$ and $f_{p}$ : the former gives high responses just for trees whereas the latter also considers small urban components (e.g. cars, fences, roof superstructures) and, more moderately, roof edges. Note also how the points corresponding to trees and facades are correctly labeled as vegetation and clutter respectively.

- Scatter $f_{s}$ measures the local height dispersion of the points. It provides a high value in the case of trees and also some undesirable urban components. This feature is usually defined as the minimal principal curvature mean of the considered point and its neighbors (Toshev et al., 2010). In the case of point sets generated from full waveform topographic Lidar systems, an alternative way to compute the scatter attribute $f_{s}$ is considered using the echo number information (Mallet and Bretar, 2009). The feature $f_{s}$ is then defined as the ratio between the number of neighbors whose echo number is strictly superior to 1 and the total number of neighbors. This alternative allows the improvement of the feature accuracy (see Section 6).

- Regular grouping $f_{g}$ is dedicated to outliers and undesirable urban components having a linear structure such as wires, facade parts, cranes or fences. This feature corresponds to the quadratic distance between the considered point and the optimal 3D-line computed among its neighbors, weighted by the number of neighbors. The response is low in the case of small isolated sets of points and linear layouts of points.

In order to tune the sensitivity of each feature, four parameters $\sigma_{e}, \sigma_{p}, \sigma_{s}$ and $\sigma_{g}$ are introduced. The features are then normalized by a linear projection on the interval $[0,1]$ of the form

$f . \leftarrow \min \left(1, \frac{f .}{\sigma}\right)$

Fig. 2 shows the behavior of these features on a small area, and underlines their complementarity in order to discriminate our four classes of interest. For example, the building roofs can be distinguished from the other urban elements as the areas having a high response to the elevation feature $f_{e}$ while having low responses to the scatter and local non-planarity features, $f_{s}$ and $f_{p}$.

\subsection{Non-supervised classification formulation}

An energy minimization is proposed to classify the point set. Let $x=\left(x_{i}\right)_{i=1 . . N_{c}}$ be a potential classification result with $N_{c}$ the number of points of the cloud, and $x_{i} \in\{$ building, vegetation, ground, clutter $\}$ the class of the $i^{\text {th }}$ point. The energy $E(x)$ is defined as a sum of partial data terms $E_{d i}\left(x_{i}\right)$ and pairwise interactions defined by the standard Potts model (Li, 2001) which introduces spatial coherence between neighboring elements:

$$
E(x)=\sum_{i=1 . . N_{c}} E_{d i}\left(x_{i}\right)+\gamma \sum_{i \sim j} \mathbb{1}_{\left\{x_{i} \neq x_{j}\right\}}
$$

where $\gamma>0$ is the parameter of the Potts model, $i \sim j$ represents the pairs of neighboring points, and $\mathbb{1}_{\{.\}}$, the characteristic function. The partial data term $E_{d i}\left(x_{i}\right)$ measures the coherence of the class $x_{i}$ at the $i^{\text {th }}$ point. It is defined as a combination of the normalized features defined above given by

$E_{d i}\left(x_{i}\right)= \begin{cases}\left(1-f_{e}\right) \cdot f_{p} \cdot f_{s} & \text { if } x_{i}=\text { building } \\ \left(1-f_{e}\right) \cdot\left(1-f_{p}\right) \cdot\left(1-f_{s}\right) & \text { if } x_{i}=\text { vegetation } \\ f_{e} \cdot f_{p} \cdot f_{s} & \text { if } x_{i}=\text { ground } \\ \left(1-f_{p}\right) \cdot f_{s} \cdot f_{g} & \text { if } x_{i}=\text { clutter }\end{cases}$ 
A Graph-Cut based algorithm (Boykov et al., 2001) is used to quickly reach an approximate solution close to the global optimum of our energy. One can easily check that our model fits the requirements for this algorithm. In our experiments, the initial configuration is chosen as the configuration minimizing the partial data terms. The energy has five parameters: $\gamma, \sigma_{e}, \sigma_{p}, \sigma_{s}$ and $\sigma_{g}$. The parameter $\gamma$ which balances the Potts interaction with respect to the partial data terms, is set to $(2 . \widehat{p})^{-1}$ where $\widehat{p}$ is the average point surface density of the dataset with respect to the XY-components. $\sigma_{e}$ is set to $6 \mathrm{~m}$ (i.e. the height of two floors), $\sigma_{s}$ to $0.5, \sigma_{p}$ to $0.5 \mathrm{~m}$, and $\sigma_{g}$ to $0.25 \mathrm{~m}$. One can imagine tuning these parameters using a learning procedure, as for example in the works of Golovinskiy et al. (2009) or Munoz et al. (2009). However, we notice that these values are stable on a wide range of input data. Thus, this would unnecessarily make the system heavier.

\subsection{Comments}

The energy model has a relatively simple formulation and provides convincing results in practice. Fig. 3 shows the potential of the model on two difficult examples, in particular, with the retrieval of two thin towers in the middle of a dense wood. Note also that the eventual local errors do not necessarily have consequences on the final result. In fact, they can be corrected during the planimetric arrangement procedure detailed later in Section 4 by using urban structure layout considerations.
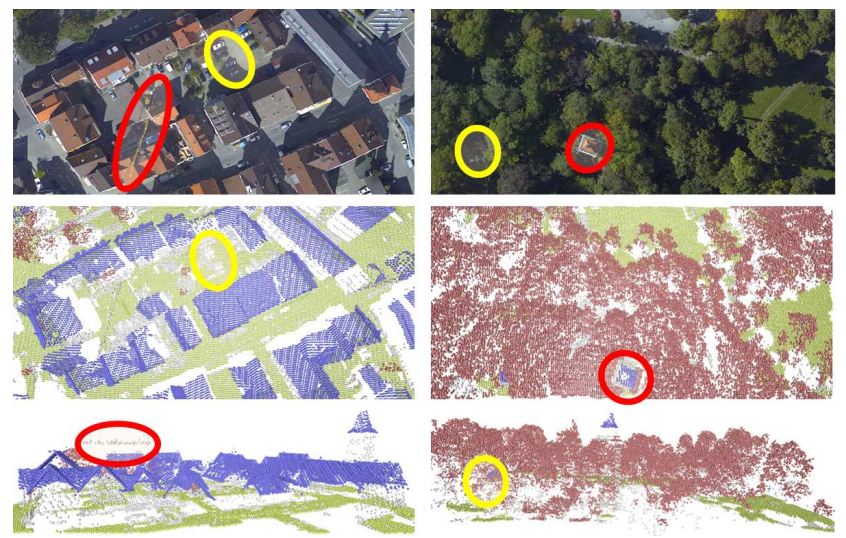

Fig. 3 Point cloud classification on two small areas - (top) aerial pictures, and (middle) top and (bottom) profile views and of the classified cloud [color code: blue=building, red=vegetation, yellow $=$ ground and white $=$ clutter $]$. Note that the two towers in the middle of a dense vegetation are correctly detected as building despite their small size (right) and how the crane, the cars, the facades and the outliers are well classified as clutter (left).

\section{Geometric shape extraction}

The second step consists in extracting 3D-primitives from the point set classified as building. As the classification proposed in Section 2 rejects outliers from the building point set, the use of Ransac-based algorithms, which are more computationally expensive for similar problems (Schnabel et al., 2007; Toshev et al., 2010), is not required. Two types of elements are detected: 3D-line segments to locate the building contours, and surface primitives to identify the roof sections. In order to be fitted by a 3D-line segment or a surface primitive, a subset of points has to verify the two following requirements:

- Minimum quality of fitting: the quadratic error $\epsilon$ between the set of the considered points and a 3D-line segment (respectively a surface primitive) is required to be inferior to a reference error $\epsilon_{s}$ (resp. $\epsilon_{a}$ ). The quadratic error $\epsilon$ between a subset of points $\left(p_{k}\right)_{k=1 . . K}$ and a manifold $\mathcal{M}$ is defined by

$\epsilon=\sqrt{\frac{1}{K} \sum_{k=1}^{K} d\left(p_{k}, \mathcal{M}\right)^{2}}$

where $d\left(p_{k}, S\right)$ is the Euclidean distance from the point $p_{k}$ to the manifold $\mathcal{M}$.

- Minimum number of points: for each primitive, a minimun number of matched points is imposed in order to guaranty robust fittings and to exclude non-significant small structures. The number of points fitted by a 3Dline segment (respectively by a surface primitive) has to be superior to a certain parameter $N_{s}\left(\right.$ resp. $N_{a}$ ) whose value is fixed according to the input data characteristics (see Section 6).

\subsection{D-line segments}

Segments are used to locate the building contours. Our concern is not to describe the contour of a building as a set of perfectly connected segments (which is a difficult task requiring urban assumptions and geometric approximations), but rather to have an accurate positioning of the main edges with potentially small parts missing between them (see Fig. 4). Indeed, our strategy consists in filling in the eventual missing parts further in Section 4 during the planimetric arrangement procedure.

First, the points located on the building borders are selected from the point set classified as building. The selection is performed by testing whether the Euclidean distance of the considered point to the optimal 3D-line 
among its neighbors is inferior to a certain threshold which depends on the point density of the input data. Indeed the spatial distribution of the neighbors of a building border point roughly describes a thin semielliptic shape, close to a 3D-line. In practice, the threshold is equal to $(2 \sqrt{\widehat{\hat{p}}})^{-1}$.

Then, 3D-lines are detected from the selected points by a clustering procedure. The process finds successive clusters of points whose quadratic error to the optimal 3D-line is inferior to $\epsilon_{s}$ and whose the number is superior to $N_{s}$. Note that the point aggregation is performed among the neighbors of the points already contained in the cluster. It allows us to detect a 3D-line formed by a compact set of points without holes. The 3D-line segments are finally obtained by projecting the two extreme points of each cluster on the corresponding optimal 3D-line.

\subsection{Planar, spherical, cylindrical, and conoidal shapes}

The surface primitives allow the detection of the regular roof sections.

The planar structures, which constitute the most common shape of roofs, are extracted first. A region growing allows the fast detection of 3D-planes. The propagation criterion tests whether the direction of the normal of the considered point is similar to those of the points in the region. When the propagation stops, the optimal 3Dplane is computed from the points of the region. The plane is then selected as a primitive if both the number of points in the region is superior to $N_{p}$ and the quadratic distance to the points of the region is inferior to $\epsilon_{p}$. This procedure is iteratively performed on the unfitted points.

Non-planar shapes are then detected from the points which have not been fitted by a plane. Extracting spheres, cylinders or cones has no obvious solution when the points only represent an unknown portion of the whole shape. One can use Monte Carlo sampling but it requires high computing time (Han et al., 2004). We prefer extracting these non-planar primitives using an iterative non-linear minimization, typically by a LevenbergMarquardt optimization. The parametrization and the first order Euclidean distance approximation to spheres, cylinders and cones proposed by Marshall et al. (2001) are used to achieve numerically stable fittings. The extracted primitives are kept if the conditions on the minimal number of points per primitive and the maximum quadratic error are validated.

Extracting non-planar shapes subsequently to the 3Dplanes avoids both high computing times and typical confusions between large non-planar primitives and planes which could have the same fitting error.

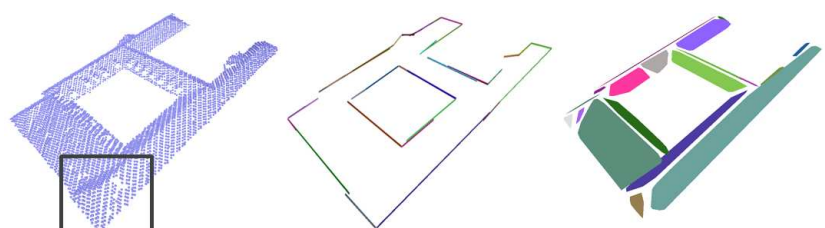

(b)

(c)

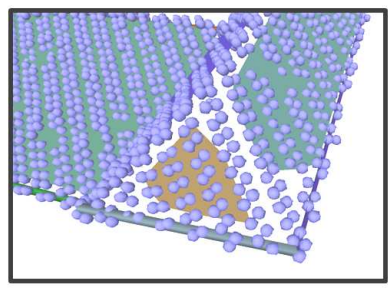

(d) (e)

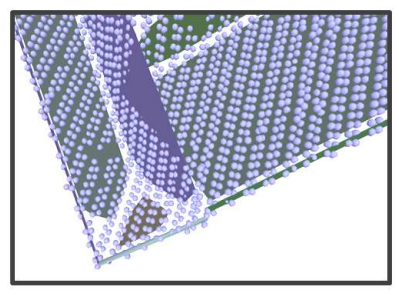

Fig. 4 Shape extraction from the building of Fig. 2 - Both (b) 3D-line segments and (c) surface primitives are extracted from (a) the set of points classified as building. The main regular roof sections of the buildings are detected as well as the global building contours. The cropped part with $(d)$ top and (e) bottom views show the primitives in the middle of the point set. Note that the planes are visually represented by their convex envelopes.

\section{Planimetric arrangement}

The third step represents the key part of the system. It consists in arranging both the geometric shapes extracted in Section 3 and the other urban components identified in Section 2 in a common dense representation. Several efficient methods of roof section arrangement have been proposed in restricted contexts. A model for planar sections is presented by Baillard and Zisserman (1999) for simple houses. Revolution sections are also taken into account by Zebedin et al. (2008), but this graph-cut based approach does not address the building contouring problem and requires building masks as input. It remains an open issue when (i) the primitives are unspecified, (ii) different types of urban objects interact in the scene, and (iii) the building contours are not given. We propose an original solution by propagating the point labels in a grid of $\mathrm{X}$ and $\mathrm{Y}$ axis under structure layout constraints (see Fig. 5). Performing the arrangement on such a grid, called a planimetric map in the following, allows us to substantially reduce the problem complexity by assuming a $2.5 \mathrm{D}$ representation of urban scenes, and also to combine two different types of 3D-geometry tools, i.e. primitives and mesh patches, in a common framework.

\subsection{Point labels and 2D-grid}

Each point of the cloud is associated with the label ground, vegetation, clutter, plane ${ }^{(l)}$, cylinder $^{(m)}$, sphere $^{(n)}$, 


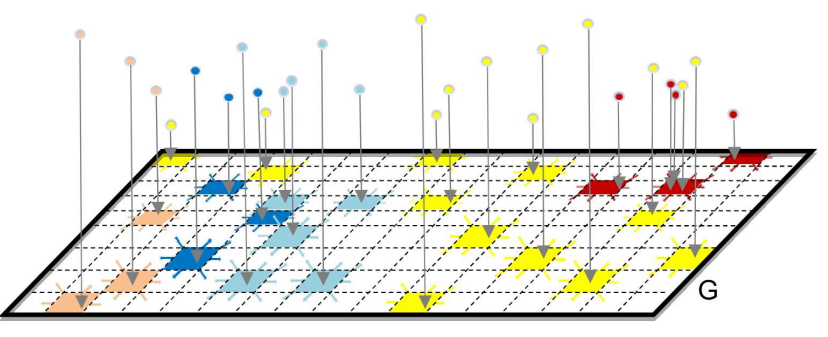

Fig. 5 The labels of the 3D-points are first projected onto a $2 \mathrm{D}$-grid $G$, and then propagated under arrangement constraints.

cone $^{(o)}$ or roof. The points labeled as clutter are not taken into account in the following. The label roof corresponds to the points classified as building in Section 2 , which have not been fitted to planar, spherical, cylindrical or conoidal shapes.

The labels of the 3D-points are projected on a 2D-grid $G$ as illustrated on Fig. 5. We denote by $G^{(p r o j)}$, the subset of $G$ composed of the cells on which at least one point label has been projected, and $G^{(e m p t y)}$, the complementary subset of $G^{(\text {proj) }}$ on $G$, i.e. the subset composed of the empty cells:

$G=G^{(p r o j)} \cup G^{(e m p t y)}$

under the condition $G^{(\text {proj })} \cap G^{(\text {empty })}=\emptyset$.

Then, the projected labels are extended to the empty cells of $G^{(\text {empty) }}$ by a basic isotropic diffusion in order to have a dense labeling on the entire grid $G$, as illustrated in Fig. 8, second column. This first label map, denoted by $l^{(i n i)}$, constitues the initial configuration of the propagation process under smoothness and structure arrangement constraints described in the next part.

\subsection{Label propagation under geometric constraints}

The label propagation procedure is performed using a Markov Random Field (MRF) with pairwise interactions, whose sites are specified by the cells of the 2D-grid $G$, and whose adjacency set $E$ is given by a breakline-dependent neighborhood. $l=\left(l_{i}\right)_{i \in G} \in L$ represents a configuration of labels of the MRF, where $L$ is the configuration space:

$L=\left\{\right.$ ground, vegetation, plane $^{(l)}, \operatorname{cylinder}^{(m)}$, sphere $^{(n)}$, cone $^{(o)}$, roof $\}^{\operatorname{card}(G)}$

The quality of a configuration $l$ is measured by the energy $U$ of the standard form:

$U(l)=\sum_{i \in G} D_{i}\left(l_{i}\right)+\beta \sum_{\{i, j\} \in E} V_{i j}\left(l_{i}, l_{j}\right)$ where $D_{i}$ and $V_{i j}$ constitute the data term and propagation constraints respectively, balanced by the parameter $\beta>0$.

\subsection{Breakline-dependent neighborhood}

The neighborhood relationship is not defined by an isotropic area, but takes into account the 3D-line segments extracted in Section 3 in order to stop the propagation beyond building contours. It is given by:

$\{i, j\} \in E \Leftrightarrow\left\{\begin{array}{l}\|i-j\|_{2} \leq r \\ \mathcal{O}\left(i, \mathcal{L}_{k}\right)=\mathcal{O}\left(j, \mathcal{L}_{k}\right)\end{array}\right.$

where $\mathcal{L}_{k}$ is the $2 \mathrm{D}$-line obtained by projecting the $k^{t h}$ $3 \mathrm{D}$-line segment interacting with the pair $\{i, j\}$ (see Fig. $6)$. $\mathcal{O}(i, \mathcal{L})$ is the oriented side in which the cell $i$ is located with respect to the line $\mathcal{L}$, and $r$ is the maximal distance between two neighboring cells. This breaklinedependent neighborhood allows us to efficiently address the building contouring problem, which is usually a critical point in existing methods.

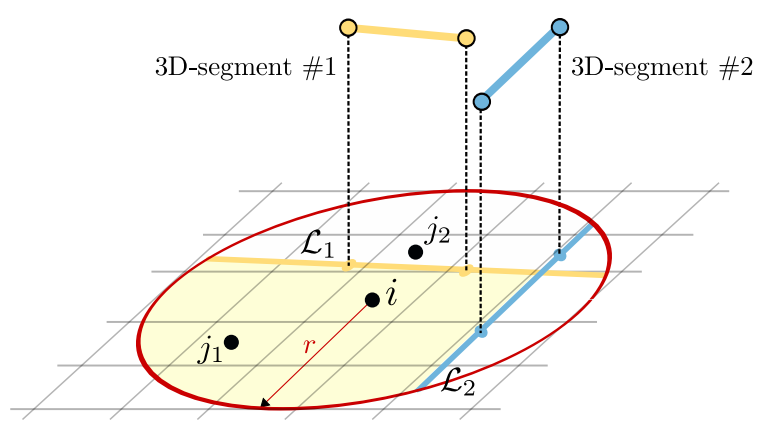

Fig. 6 Breakline-dependent neighborhood- The neighbors of the cell $i$ are contained in the yellow area. $\left\{i, j_{1}\right\} \in E$ but $\left\{i, j_{2}\right\} \notin E$. Note that the 3D-line segments do not have to be connected as the yellow area is computed by intersecting the 2D-lines supporting the segments.

\subsection{Data term}

The term $D_{i}$ checks the coherence of the label $l_{i}$ at the cell $i$ with respect to the input point cloud. The term is given by

$D_{i}\left(l_{i}\right)= \begin{cases}c & \text { if } l_{i}=\text { roof } \\ \min \left(1,\left|z_{l_{i}}-z_{p_{i}}\right|\right) & \text { else if } i \in G^{(\text {proj })} \\ 0 & \text { otherwise }\end{cases}$

where $c \in[0,1]$ is a coefficient penalizing the labels roof in order to favor the primitive-based description 
of buildings. $z_{l_{i}}$ is the height associated with $l_{i}$, and $z_{p_{i}}$ the maximal height of the input 3D-points contained in the cell $i$.

\subsection{Propagation constraints}

The potential $V_{i j}$ allows both the label smoothness and a coherent arrangement of the structures. To do so, an arrangement law, denoted by $\bowtie$, is introduced to test whether two labels, $l_{i}$ and $l_{j}$, of neighboring cells, $i$ and $j$, are spatially coherent:

$l_{i} \bowtie l_{j} \Leftrightarrow \mathcal{O}\left(i, \mathcal{I}_{l_{i}, l_{j}}\right) \neq \mathcal{O}\left(j, \mathcal{I}_{l_{i}, l_{j}}\right)$

where $\mathcal{I}_{l_{i}, l_{j}}$ is the $\mathrm{XY}$-intersection between the two objects $l_{i}$ and $l_{j}$, and $\mathcal{O}(i, \mathcal{I})$ is the oriented side in which the cell $i$ is located with respect to the curve $\mathcal{I}$. In other words, the intersection of the two objects must be spatially located in between the two cells $i$ and $j$.

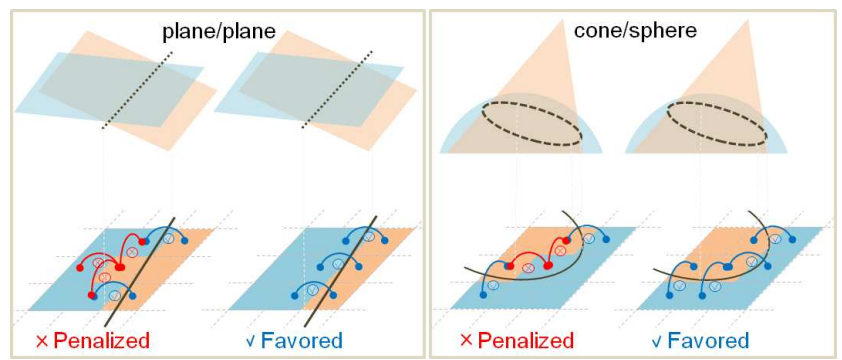

Fig. 7 Principle of the $\bowtie$-law on two examples - The blue (respectively red) junctions between neighboring cells correspond to spatially coherent (resp. non-coherent) labels.

For example, if two neighboring cells are associated with two different planar labels, the $\bowtie$-law will check that the projection in the $2 \mathrm{D}$-grid of the $3 \mathrm{D}$-line intersecting the two 3D-planes is located in between the two cells. Thus, the exact separation of two connected planes is constrained as illustrated in Fig. 7.

Finally the pairwise interaction is formulated by:

$V_{i j}\left(l_{i}, l_{j}\right)=\left\{\begin{array}{l}\epsilon_{1} \text { if } l_{i} \bowtie l_{j} \\ \epsilon_{2} \text { if } l_{i}=l_{j} \\ 1 \text { otherwise }\end{array}\right.$

where $\epsilon_{1}$ and $\epsilon_{2}$ are real values in $[0,1]$ with $\epsilon_{1}<\epsilon_{2}$. They tune the label smoothness with respect to the coherent object arrangement considerations.

\subsection{Optimization with parallelization scheme}

Finding the label configuration which minimizes the energy $U$ is a non-convex optimization problem. Simulated annealing techniques (Li, 2001), graph-cut based algorithms e.g. (Boykov et al., 2001) or belief propagation methods e.g. (Weiss and Freeman, 2001) could provide a good approximation of the solution but at the expense of high computing time. The scenes are generally of a large scale and the number of labels is very high.

In order to reach reasonable computing times, an original parallelization scheme is proposed, relying on the two following assumptions:

- H1: the labels cannot be propagated between two nonoverlapped urban objects in the scene (e.g. the label corresponding to the roof section of a building cannot be used for another building),

- H2: the point labels originally projected in the grid $G^{(p r o j)}$ are of quality, i.e. they are probably correct (See Fig. 5).

The grid $G$ is partitioned in an unknown number $N$ of clusters such that

$G=\cup_{k \in[1, N]} G_{k}$

with $G_{k} \cap G_{k^{\prime}}=\emptyset, 1 \leq k<k^{\prime} \leq N$. The partition is obtained from the initial label map $l^{(i n i)}$ by separating the low-level urban components (e.g. blocks of buildings and groups of trees) which are supposed to be independent of each others (H1). The quality of the partition relies on the initial label map $l^{(i n i)}$, and thus on the point labels originally projected in the $2 \mathrm{D}$-grid (H2).

Each cluster $G_{k}$ corresponds to a set of connected cells labeled as non-ground in the initial label map $l^{(i n i)}$, and whose area is maximal. In particular, it implies that the outside contour of $G_{k}$, denoted by $\partial G_{k}$, is labeled as ground:

$\forall i \in \partial G_{k}, k \in[1, N-1], l_{i}=$ ground

Note that a morphological erosion is preliminarily performed in the initial label map on the cells labeled as ground to give robustness to the component separation and avoid the omission of building pieces. As illustrated on Fig. 9, the last cluster $G_{N}$ corresponds to the remaining cells labeled as ground. Fig. 14 also shows an example of a grid partitioning on a $1 \mathrm{~km}^{2}$ dense urban area.

The original configuration space $L$ (see Eq. 6) can be then significantly reduced by decomposing the minimization of $U$ as a set of $N-1$ local independent (and thus parallelizable) energy minimization problems over 


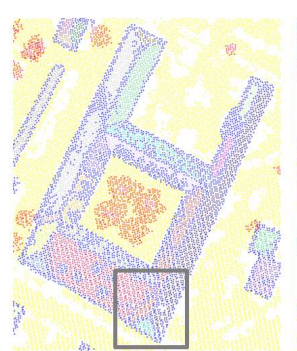

(a)
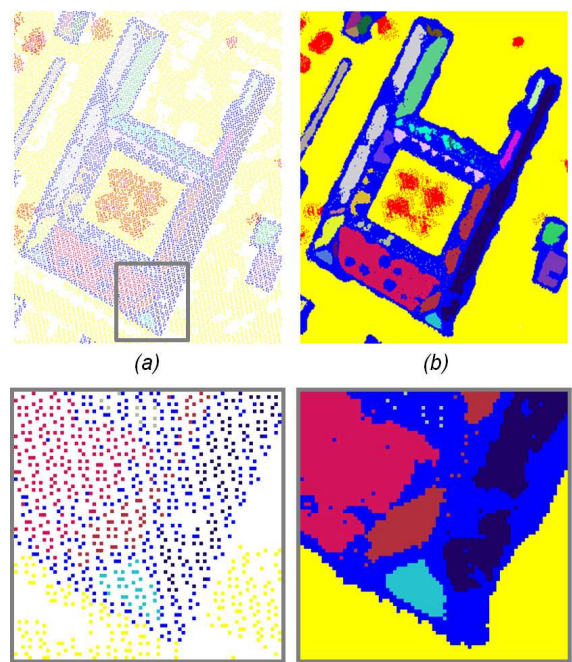

(b)

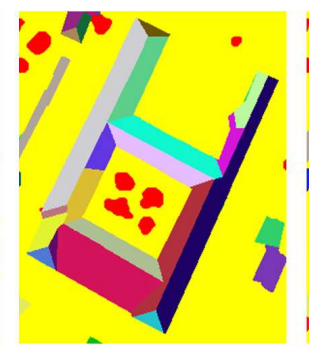

(c)
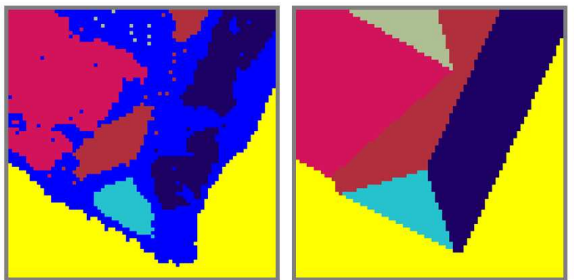

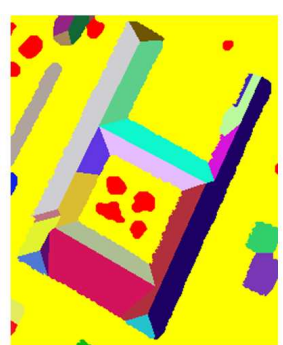

(d)

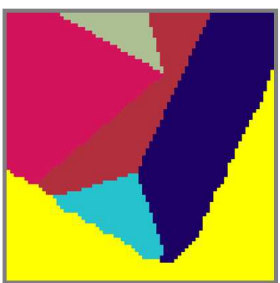

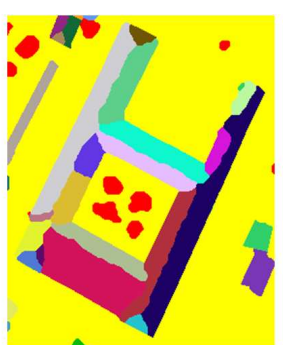

(e)

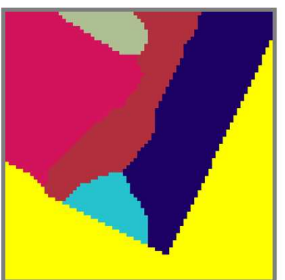

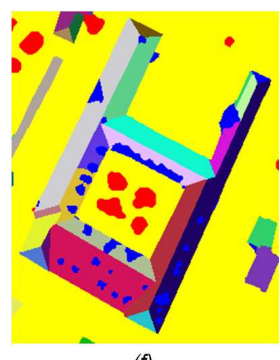

(f)

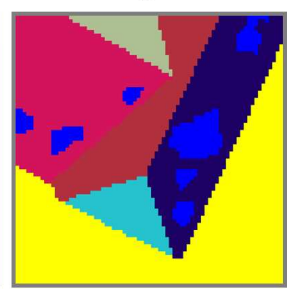

Fig. 8 Planimetric arrangement from the building of Fig. 2 - (a) the grid $G^{(p r o j)}$ of the projected point labels, (b) the initial label map $l^{(i n i)},(c)$ the label map after minimizing $\mathrm{U},(d)$ the label map after minimizing a variant of $\mathrm{U}$ where the breakline-dependent neighborhood is substituted by a standard isotropic neighborhood, $(e)$ the label map after minimizing a variant of $U$ where the $\bowtie$-law is not taken into account, and $(f)$ the label map after minimizing $U$ whose parameter $c$ has been significantly decreased. One can notice that the label propagation is correctly stopped beyond building contours and neighboring primitives. The $\bowtie$-law allows the optimal arrangement of the roof sections, and the breakline-dependent neighborhood avoids the wavy building contours. Note also how the decrease of the parameter $c$ impacts on the apparition of roof labels in order to describe the small irregular roof components [color code: white=empty cell, yellow=ground, red=vegetation, blue=roof, other colors=primitives].

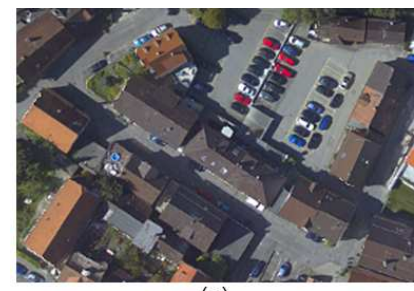

(a)
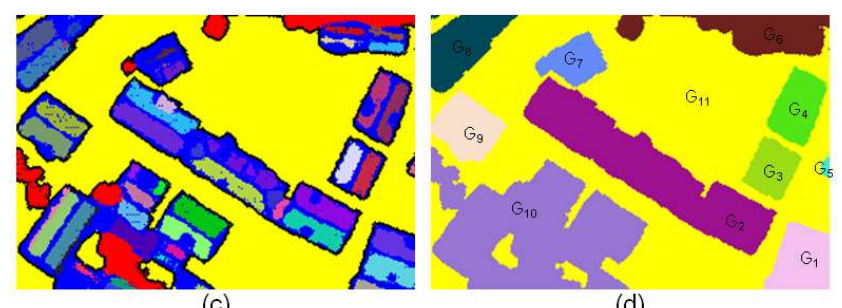

(c)

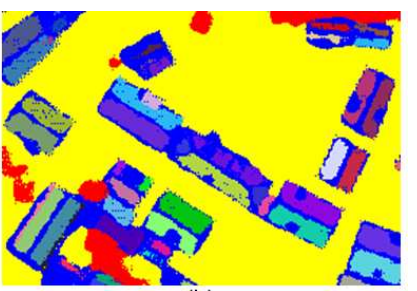

(b)

(d)

Fig. 9 Partitioning of the grid on a downtown sample - (a) an aerial picture of the scene (not used), (b) the initial label map $l^{(i n i)},(c)$ the initial label map $l^{(i n i)}$ with a morphological erosion performed on the cells labeled as ground and illustrated by the black contours, and $(d)$ the resulting partition where each of the 11 clusters is illustrated by a random color. The last cluster $G_{11}$ corresponds to the eroded set of ground cells.

the partition of the grid $G$ :

$\min _{l \in L} U(l) \Leftrightarrow\left\{\begin{array}{l}\min _{l / G_{k} \in L_{k}} U\left(l_{/ G_{k}}\right), \forall k \in[1, N-1] \\ l_{/ G_{N}}=\{\text { ground }\} \operatorname{card}\left(G_{N}\right)\end{array}\right.$ where $l_{/ G_{k}}$ is a configuration of labels on the cluster $G_{k}$, and $L_{k}$ the local configuration space on the cluster $G_{k}$. In order to limit the number of possible labels per local problem, $L_{k}$ only contains the labels present in $l_{/ G_{k}}^{(i n i)}$ :

$L_{k}=\left\{l_{i} / l_{i} \in l_{/ G_{k}}^{(i n i)}\right\}^{\operatorname{card}\left(G_{k}\right)}$

Thus the label of a primitive belonging to a certain cluster is not uselessly tested in another cluster (H1). This decomposition scheme has also another advantage: the last cluster $G_{N}$ of the remaining cells labeled as ground, which is usually of big size, is not concerned by the optimization. We rely here on the hypothesis $\mathrm{H} 2$ which allows a significant gain of time.

Table 1 Comparisons of different optimization techniques on a $1 \mathrm{~km}^{2}$ dense urban area.

\begin{tabular}{|c|c|c|}
\hline & Energy & Time \\
\hline $\begin{array}{c}\alpha \text {-expansion } \\
\text { (Boykov et al., 2001) }\end{array}$ & 2832.9 & $402 \mathrm{~min}$ \\
\hline $\begin{array}{c}\text { Belief propagation } \\
\text { (Weiss and Freeman, 2001) }\end{array}$ & 3016.6 & $618 \mathrm{~min}$ \\
\hline $\begin{array}{c}\alpha \text {-expansion with our } \\
\text { parallelization scheme }\end{array}$ & 2853.3 & $3.5 \mathrm{~min}$ \\
\hline
\end{tabular}

The $\alpha$-expansion algorithm (Boykov et al., 2001) is used to solve each local independent optimization problem. This algorithm is particularly efficient in our context, 
i.e. with a limited number of labels and a good initial configuration. Confidence is given to the labels originally projected: the expansions are first performed on the subset $G^{(e m p t y)}$, i.e. the cells originally considered as empty, and then on the complementary subset $G^{(p r o j)}$ to readjust the configuration.

As shown in Tab. 1, the parallelization scheme allows us to reach a good approximation of the solution while significantly reducing the computing times on an 8-core computer compared to standard techniques. The energy reached by our parallelization optimization scheme is slightly higher than by the conventional $\alpha$-expansion minimizing the global energy problem. Indeed, the clustering procedure enforces exterior borders of clusters to be labeled as ground. Some local errors can then be generated when the clusters are not ideally separated, in particular when exterior borders of clusters overlap with some building components. By enlarging the structure element of the morphological erosion, this type of errors decreases but the computation time is increased as the number of clusters is reduced.

\section{Representation of the urban elements}

The three types of elements contained in the scenes are differently represented in 3D from the obtained label map. Buildings are modeled by combining arrangements of geometric 3D-primitives and mesh patches, trees by template matching, and the ground by a meshing procedure guarantying a continuous surface.

\subsection{Buildings}

A hybrid representation is used to model the buildings with a high level of generalization and a good compaction. Arrangements of geometric 3D-primitives for the standard roof sections, and mesh-patches describing the irregular roof components are combined.

The primitive arrangements are represented by polyhedral structures extracted from the label map obtained in Section 4. The primitive intersections are directly computed from the primitive adjacency graph given by the label map. Note that, in case of non-planar primitives such as spheres or cylinders, the geometric accuracy of the polyhedral structure is fixed by a discretization parameter.

The mesh-patches are initially created by triangulating the cells labeled as roof. A Z-component is associated to the XY-center of these cells so that triangular meshes are directly obtained from the 8-connexity structure of cells. The Z-component of the cell $i$ is taken as the maximal height $z_{p_{i}}$ of the input 3D-points contained in the cell $i$ if $i \in G^{(p r o j)}$, and as the mean height of the closest input 3D-points otherwise. As illustrated on Fig. 10, one of the main advantages of this strategy is the simplification of the mesh-patches while controlling the approximation error. A standard mesh simplification algorithm (Garland and Heckbert, 1997) can then be used to obtain more compact and coarser building representations.

The facades are obtained by projecting vertically the building contours on the estimated ground. The final result can be seen as a general triangular mesh in which the regular roof sections associated to a planar primitive are usually represented by one or two triangular facets and some finer mesh-patches describe the irregular components, as illustrated on Fig. 12.

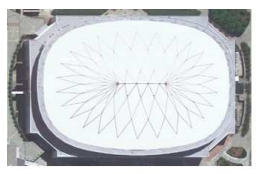

(a)

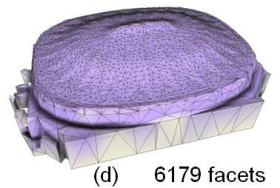

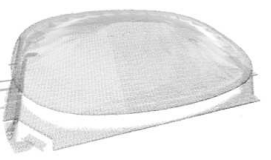

(b)

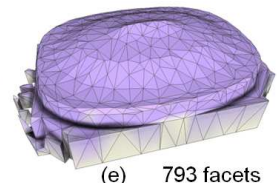

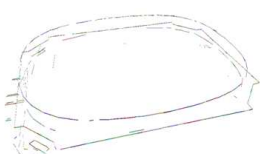

(c)

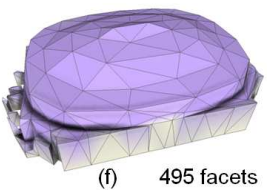

Fig. 10 Simplification of the mesh patches on an irregular roof(a) an aerial picture of the scene, (b) the input point set with a $17 \mathrm{pts} / \mathrm{m}^{2}$ density, (c) the extracted 3D-line segments, and the obtained 3D-models with $(d)$ fine, $(e)$ medium and $(f)$ coarse mesh-patches. Note that the primitive arrangement and the facades are not affected by the simplification process.

\subsection{Trees}

They are reconstructed in 3D using template matching. The template is a simple ellipsoidal tree model whose compaction and rendering are well adapted to large urban scenes (see Fig. 11). For a street-view representation, one can imagine proposing a more realistic tree modeling, e.g. (Xu et al., 2007). As directly matching an ellipsoid to the point set is computationally expensive, the center of mass of trees is first detected using a watershed algorithm performed on the estimated height of the cells labeled vegetation. The other parameters of the template such as the height and the radius of the crown are then simultaneously found by minimizing the Euclidean distance from points to an ellipse. The tree trunk is modeled by a cylinder which makes the link between the ellipsoid and the ground surface. 


\subsection{Ground}

A standard meshing procedure is used to model the ground surface by a continuous surface. A grid of 3Dpoints is created from a spatial sub-sampling of the cells labeled as ground. It allows an accurate description without imposing any geometric constraints on the surface. Note that, similar to the mesh-patches of the buildings and the non-planar primitives, the mesh can be simplified using a decimation algorithm (Garland and Heckbert, 1997) to gain in compaction as shown in Fig. 12 .
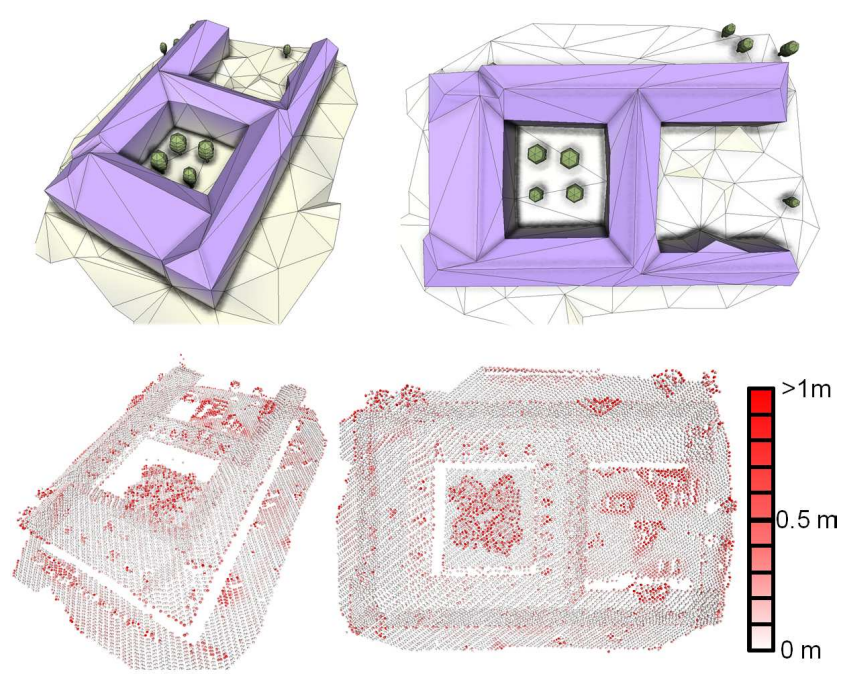

Fig. 11 Object representation - (top) obtained 3D-model and (bottom) input cloud $\left(2 \mathrm{pts} / \mathrm{m}^{2}\right.$ ) with the points colored according to their distance to the 3D-model. The high errors correspond to points from trees (the points of a tree do not obviously describe a perfect ellipsoidal shape) and from small urban components such as cars or roof superstructures. The mean error is $0.2 \mathrm{~m}$, and the number of triangular facets is 205 without including the trees. Note that the surface primitives are divided into triangular facets for visual rendering and compaction measurement.

\section{Experiments}

6.1 Implementation and parameter settings

The algorithm has been implemented in $\mathrm{C}++$ using the Computational Geometry Algorithms Library (CGAL, 2011). This library provides the basic geometric functions for the analysis of point clouds and the mesh processing. For example, this allows the search of neighbors in the input cloud or the computation of distances from point to parametric surfaces.

Several parameters are introduced during the four steps of the algorithm. One of the major strengths of the algorithm is that the point density of the input data does not interfere with the planimetric arrangement in terms of result quality and computation complexity. Thus, most of the parameters are stable on a large range of input data. The size of a cell $s_{c}$ is usually chosen in the interval $[0.2 \mathrm{~m}, 0.4 \mathrm{~m}]$. The radius $r$ of the breakline-dependent neighborhood is fixed to $1.5 s_{c}$. The parameters of the pairwise interactions in the planimetric arrangement model proposed in Eq. 11 are chosen as $\epsilon_{1}=0.5 \times \epsilon_{2}=\frac{1}{3}$ and $\beta=0.5$.

Other parameters depend on the input data types as shown in Tab. 2. This concerns the primitive extraction parameters, i.e. $N_{s}, N_{p}, \epsilon_{s}$ and $\epsilon_{p}$ which are sensitive to the point density of the input cloud and also to the acquisition type (laser or MVS). The number of expansion cycles during the optimization of the label map (see Section 4.2) has also to be set according to the point density of the input data. More precisely, it must be set according to the proportion of empty cells in the map: the lower this ratio, the lower the number of expansion cycles.

Table 2 Parameter settings in function of the input data type

\begin{tabular}{|c|c|c|c|c|c|}
\hline & $N_{s}$ & $N_{p}$ & $\epsilon_{s}$ & $\epsilon_{p}$ & Exp. cycles \\
\hline Lidar, $2 \mathrm{pts} / \mathrm{m}^{2}$ & 12 & 15 & 0.4 & 0.1 & 6 \\
\hline Lidar, $17 \mathrm{pts} / \mathrm{m}^{2}$ & 25 & 100 & 0.2 & 0.1 & 4 \\
\hline MVS, $16 \mathrm{pts} / \mathrm{m}^{2}$ & 15 & 120 & 0.5 & 0.5 & 4 \\
\hline MVS, $100 \mathrm{pts} / \mathrm{m}^{2}$ & 35 & 500 & 0.4 & 0.5 & 2 \\
\hline
\end{tabular}

\subsection{Visual considerations}

Our method has been tested on various types of urban landscapes. Most of the datasets has been acquired by laser, more precisely with lidar systems having different point densities from 2 and $17 \mathrm{pts} / \mathrm{m}^{2}$.

Fig. 18 shows large scenes reconstructed with different types of landscapes including business districts with large and tall buildings, historic towns with a high concentration of both small buildings and trees, and hilly areas with high altimetric variations and dense forests. The input data generated from aerial laser scanning contain more than ten million of points. The results are obtained without using prior information on the landscape type and the object distributions within the scenes.

The level of detail of the results depends mainly on the input point density. For example, the roof details such as the dormer-windows or chimneys in Fig. 11 are described by less than 4 points in the $2 \mathrm{pts} / \mathrm{m}^{2}$ density data. Our method ignores these sets of points in the 


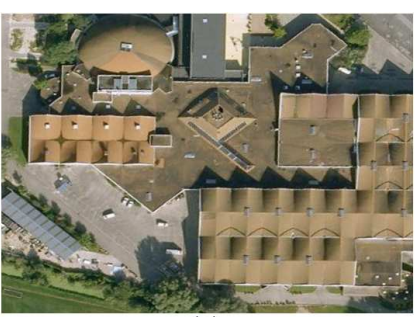

(a)

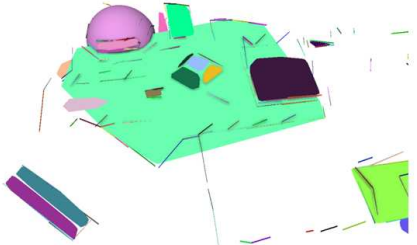

(b)

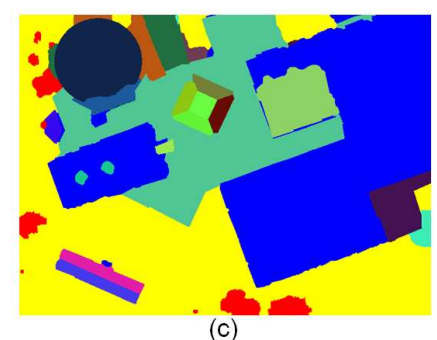

(c)

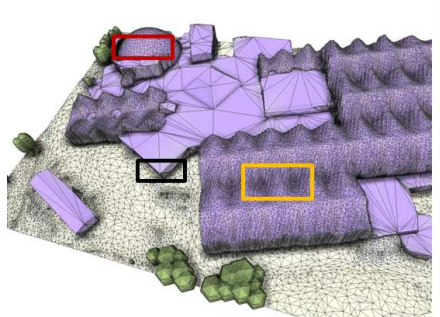

(d)

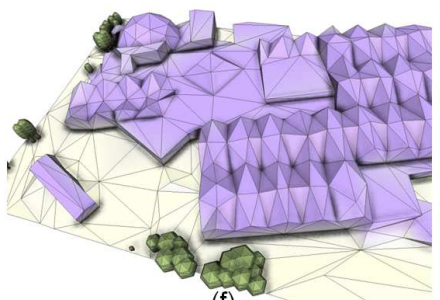

(f)
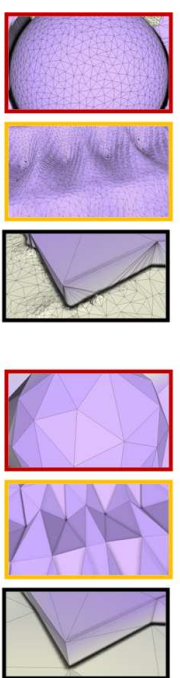

mean error (meter)

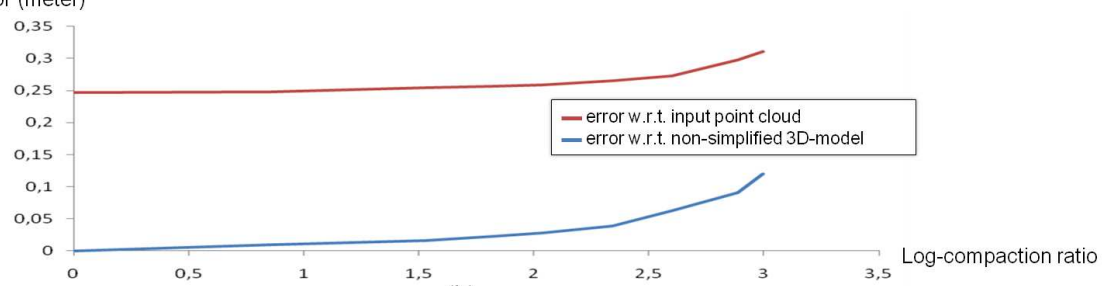

(h)

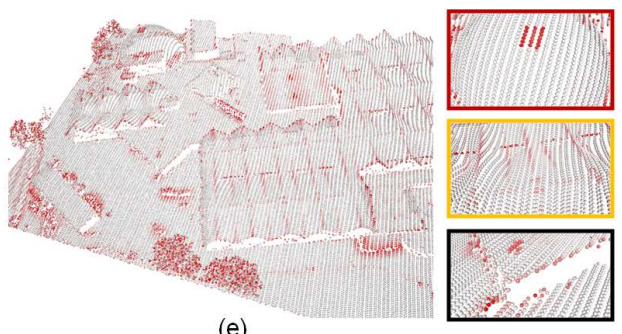

(e)

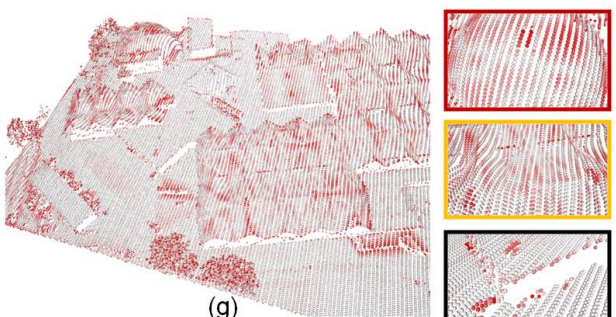

(g)

Fig. 12 Hybrid reconstruction of a complex building - (a) aerial picture, (b) extracted 3D-primitives, (c) label map [color code: see Fig. 8], 3D-models obtained with $(d)$ fine and $(f)$ coarse mesh patch descriptions, input cloud $\left(2 \mathrm{pts} / \mathrm{m}^{2}\right)$ with the points colored according to their distance to the (e) "fine" and $(g)$ "coarse" 3D-models [color code: see Fig. 11], and (h) error graph of the 3Dmodels with mesh-patch simplification w.r.t. the input point cloud (red) and the unsimplified 3D-model (blue) in function of the log-compaction ratio of the unsimplified 3D-model to the simplified one. Our hybrid representation is particularly interesting in such a case: the building is accurately modeled by planes and a sphere for the regular parts, and by mesh-patches for the atypical surfaces, i.e. the undulating roofs. The fine (respectively coarse) 3D-model has $46 K$ facets (resp. 864 facets) and a $0.24 \mathrm{~m}$ (resp. $0.33 \mathrm{~m}$ ) mean error to the input data.

computation of the main roof sections because they are too small to extract robust information. In Fig. 15, the input data has a $17 \mathrm{pts} / \mathrm{m}^{2}$ density which is high enough to recover roof details such as the chimney. The building contours are correctly located, due to the breaklinedependent neighborhood introduced in the planimetric arrangement, even when they overlap at different locations with trees as shown in Fig. 13 (Building \#2).

One of the main advantages of this hybrid representation is that the eventual primitive under-detection does not necessarily penalize the approach in terms of results. Indeed the regular roof sections missed during the geometric shape extraction stage are completed by mesh-patches. The final 3D-model remains coherent and correct even if it loses in terms of compaction. The eventual under-detection of 3D-line segments is more penalizing, especially when the input cloud has both a spatially heterogeneous point distribution and a low point density. In such a case, the building 3Dmodels can have wavy contours which correspond to the shape induced by the bordering points of the building as shown in Fig. 12. One solution can be then to simplify the mesh but this engenders a loss of accuracy. On the other hand, over-detecting primitives would increase the number of labels during the planimetric arrangement, and thus, the computing times as well as the compaction of the 3D-model.

\subsection{Performances}

The evaluation of building reconstruction methods is a difficult task due to the absence of a benchmark in the field, the problems of data sharing as well as the difficulty in achieving ground truth. In order to measure the quality of the results, two main criteria are considered: the distance of the input points to the 3D-model and the compaction of the 3D-model. The mean distance on a $2 \mathrm{pts} / \mathrm{m}^{2}$ density point cloud is typically contained in the interval $[0.2 \mathrm{~m}, 0.35 \mathrm{~m}]$ (see Fig. 11 and 12). However, the mean distance is computed from all the points 


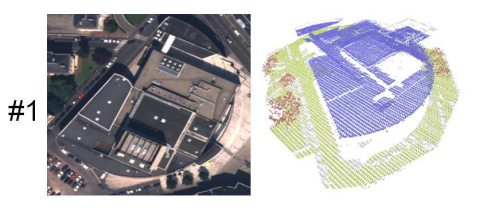

\#2

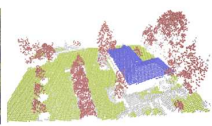

\#3
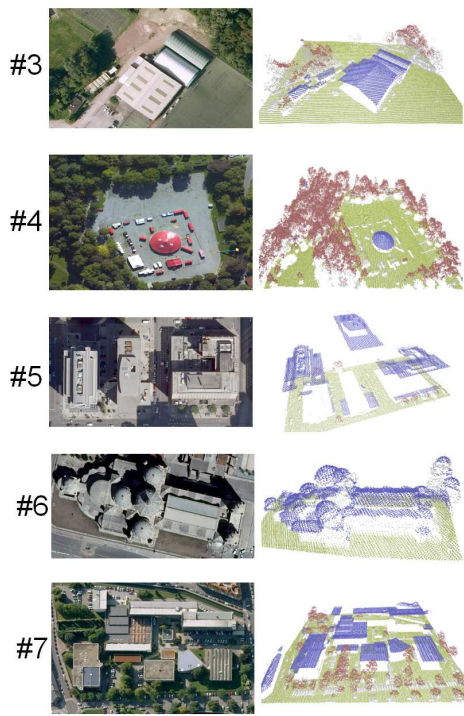

(a)

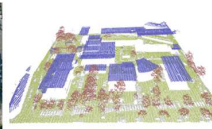

(b)
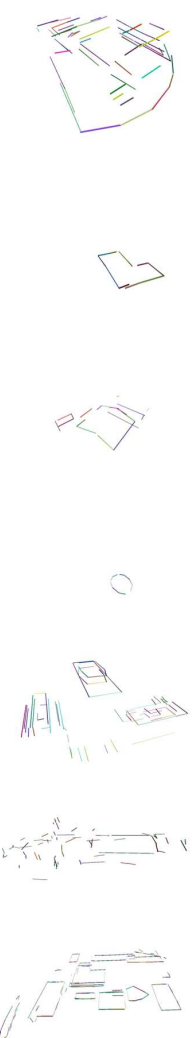

(c)
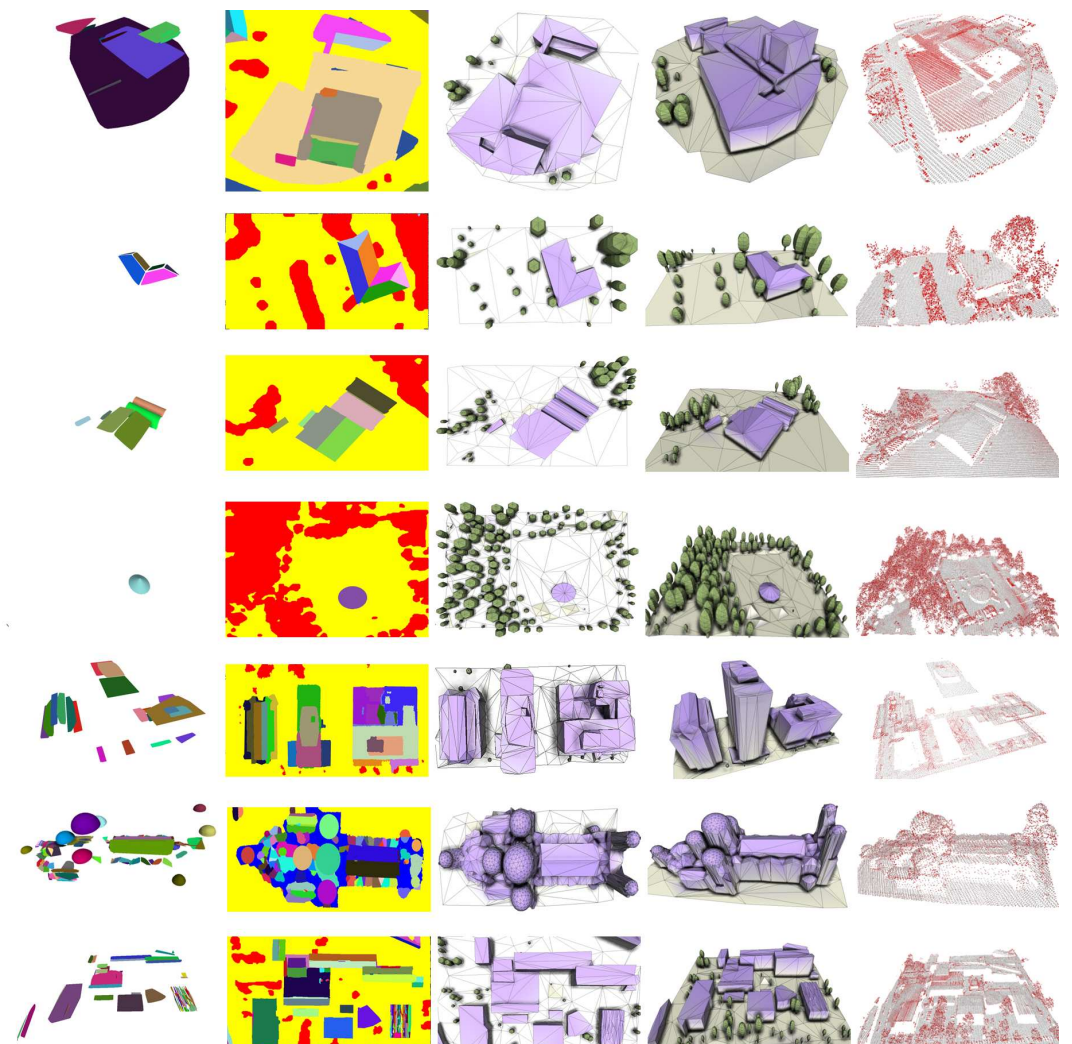

(d)

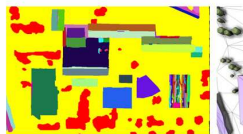

(e)

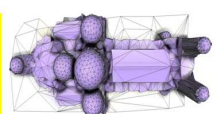

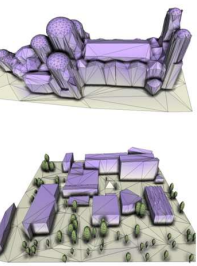

(g)

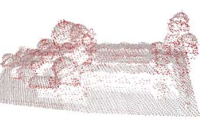

Fig. 13 Results on several buildings with varying point densities (from 2 to 5 pts $\left./ \mathrm{m}^{2}\right)$ - $(a)$ aerial pictures, $(b)$ classified point sets [color code: see Fig. 3], $(c)$ extracted 3D-line segments, $(d)$ extracted surface primitives, $(e)$ label maps, $(f)$ top and $(g)$ profile views of the obtained 3D-models, and $(h)$ input point data with the points colored according to their distance to the associated 3D-models [color code: see Fig. 11]. Building \#1 is an atypical piecewise planar structure with curved footprints. Building \#2 is a classic house surrounded by trees. Note how the building and the trees are correctly reconstructed in spite of the fact they overlap at different locations. Building \#3 is a simple structure with cylindrical parts. Building \#4 is a circus with a conoidal shape. Note how the trucks located around the circus are rejected as clutter during the point set classification. Building \#5 represents a set of three north American skyscrapers which are particularly well adapted to the Manhattan World assumption. Building \#6 is a Roman cathedral with a complex structure including spherical domes, small planar sections and irregular roof parts. Building \#7 is a typical set of industrial structures.

of the input data: this includes the outliers and the undesirable points corresponding to cars, fences or wires, which highly corrupt the obtained mean error. Without taking these points into account, the mean error is usually inferior to $0.1 \mathrm{~m}$.

We compare our method according to these two criteria to the mesh simplification algorithm proposed by Zhou and Neumann (2010). The compaction of our model is almost twice better, for a similar mean error to the input data as shown in Fig. 15.

In addition, we evaluate the altimetric accuracy of the algorithm with respect to the ground truth obtained by the topographical measurement on two buildings, and compare it to a constructive solid geometry approach and a Digital Surface Model from point cloud as shown in Fig. 16. From a $2 \mathrm{pts} / \mathrm{m}^{2}$ density input data, we obtain the best mean error, i.e. $0.21 \mathrm{~m}$, on the evaluated buildings in spite of some high local errors on the contours illustrated by the thin black lines partially surrounding the buildings on the altimetric error maps. From such a low point density, it is indeed difficult to perfectly extract the building contours.

In regard to tree detection, the results are satisfactory. The false alarm rate and the under-detection rate are respectively estimated to $2 \%$ and $6 \%$ on the Amiens dataset. However, certain building contour points associated with atypical roof sections may be detected as vegetation, especially when the scatter feature $F_{s}$ is computed without using echo information (see Fig. 18, top right crop).

Around 10 minutes is required to model a $1 \mathrm{~km}^{2}$ dense urban area using a single computer. The computing times are competitive compared to most of the large scale modeling algorithms, e.g. Poullis and You (2009) 


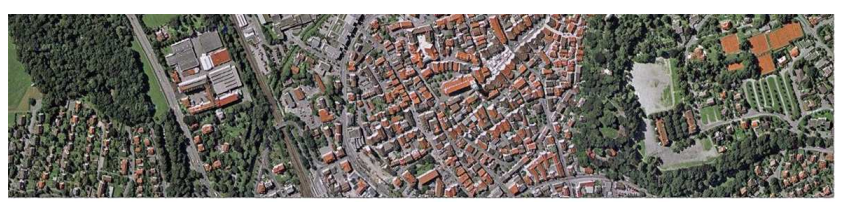

(a)
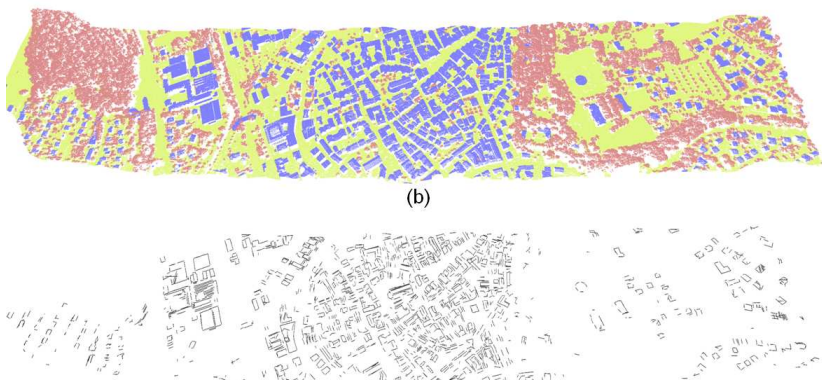

(c)

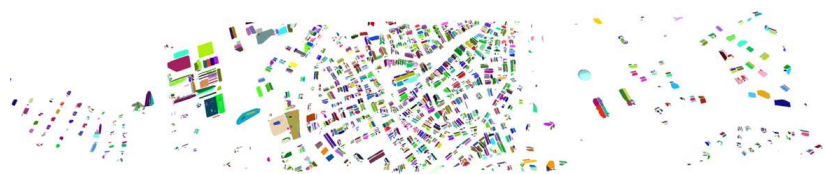

(d)
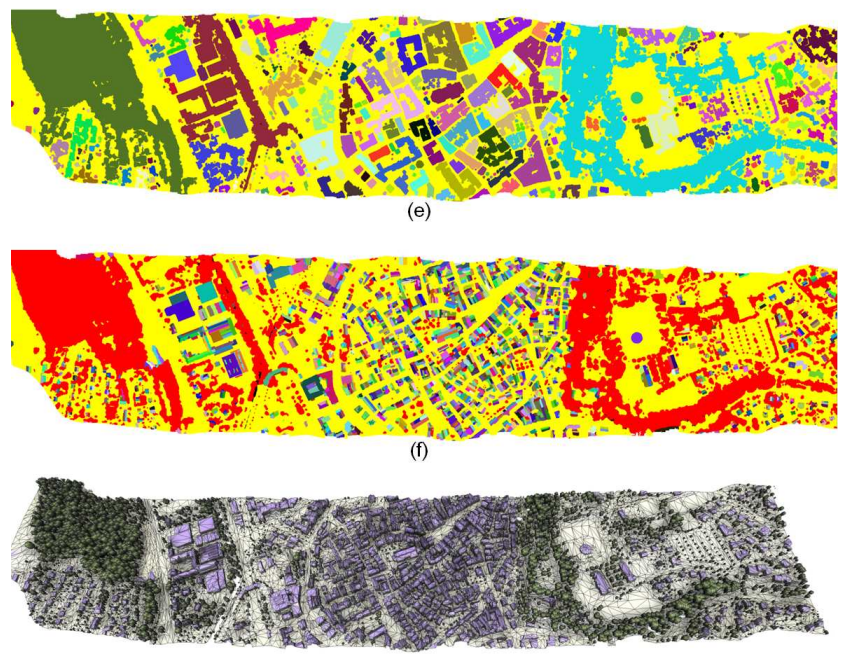

(g)

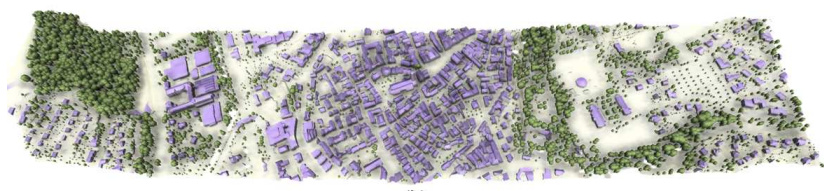

Fig. 14 Result from a 2.3M point set representing a $1 \mathrm{~km}^{2}$ area urban scene (Biberach, Germany) with a $128 \mathrm{~m}$ altimetric variation- (a) an aerial picture (not used) of a city center, (b) the classified point set [color code: see Fig. 3], (c) the extracted 3D-line segments, (d) the extracted surface primitives, $(e)$ the partition of the grid $\cup_{k \in[1, N]} G_{k}$ for the optimization decomposition [each cluster $G_{k}$ is randomly colored], $(f)$ the label map, the obtained 3D-model $(g)$ with and $(h)$ without mesh visualization. The result is obtained in approximatively 10 minutes. Note that, as the aerial picture has been captured several years before the point data, some buildings are missing on this picture.
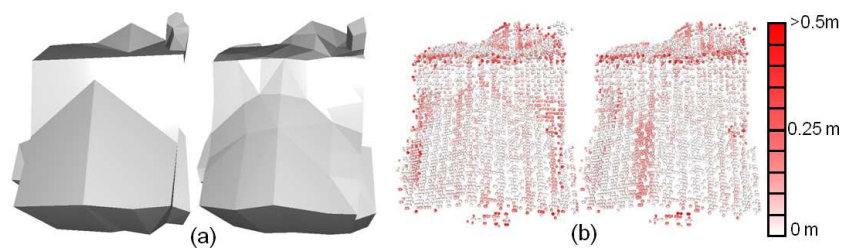

Fig. 15 Comparison with a mesh simplification method- (a) 3Dmodels obtained (left) by our method and (right) by Zhou and Neumann (2010), (b) input clouds $\left(17 \mathrm{pts} / \mathrm{m}^{2}\right)$ with the points colored according to their distance to the associated 3D-models. Our model presents a better roof component recovery. Although the mean errors to the input data are similar $(0.07 \mathrm{~m})$, the compaction of our model is almost twice better (126 vs 228 facets).

with around half an hour per $\mathrm{km}^{2}$, or Muller et al. (2006) who require several interactive operations per building.

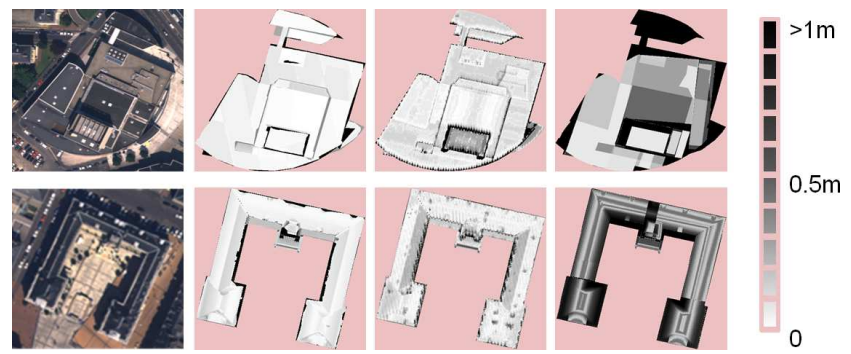

Fig. 16 Comparison with pixel-based and primitive-based approaches in terms of altimetric accuracy w.r.t. ground truth (from left to right) aerial pictures of two buildings, altimetric error maps for our method, for a Digital Surface Model from point cloud, and for the Constructive Solid Geometry approach by Lafarge et al. (2010a). Note how the roof sections from our method are accurately estimated when compared to the other algorithms.

\subsection{Point clouds from laser or MVS?}

The acquisition type of the input data impacts on the result quality provided by our method. Several works, such as the study of Leberl et al. (2010), compare the potential of laser and MVS for urban scene analysis. Such comparisons are usually difficult to realize as the performances depend strongly on the own characteristics of the acquisition system, and also on the dense stereo algorithm used to generate the DSM in case of MVS acquisition.

Contrary to the point sets from MVS, laser-based point clouds have a high altimetric accuracy but a heterogeneous planimetric distribution, and usually a lower point density. These differences play an important role during the surface primitive extraction step. As illustrated in Tab. 2, the maximal fitting errors and the 
minimal numbers of fitted points per primitive must be higher in the case of a MVS-based input data in order to compensate for the approximative altimetric accuracy of the points. In order to improve the surface primitive extraction procedure in the case of low resolution MVS-based input data, one can substitute the quadratic error (see Eq. 4) by a softer distance such as the $L_{1}$-norm error which is frequently used from MVSbased DSM computations (Xu and Zhang, 1996).

At low resolutions, the DSM-based point clouds do not have strongly marked discontinuities on the building contours as shown on the crops in Fig. 17. This is due to the dense stereo algorithms used to generate the DSM which usually introduce smoothness constraints on the surface. This point penalizes the recovery of the building contours compared to the laser acquisition.

The tree detection is more efficient from laser-based point clouds than from MVS-based data. Indeed, the point diffusion of a tree from laser has a more irregular geometry than from MVS, and is thus more discriminative for tree recognition. However, the tree detection from MVS can be easily improved by taking into account radiometric information contained in the images (e.g. texture attributes), or by using, when available, the Near InfraRed channel known to give high responses for vegetation.

Finally, our algorithm globally provides better results from laser than from MVS. 3D-models from MVS-based point sets at low resolution usually have shape approximation errors. At high resolution, the results are similar to those obtained from laser but the computation times of the first and second steps of the algorithm are higher.

\subsection{Limitations}

First, some urban components are not taken into account in our representation. In particular, the bridges and the elevated roads which are local planar structures elevated above the ground are frequently detected as buildings (see Fig. 18, top right crop). This problem can be solved by considering additional urban components in the point cloud classification. Note that in this perspective, the energy formulation of the planimetric arrangement can be easily adapted. Secondly, the modeling of the trees is restricted to the use of an ellipsoidal shape template. It is sufficient for large scene descriptions but too limited for street-view representations. In light of this, it seems relevant to introduce a library of tree forms and create more complex dependencies between neighboring elements. Thirdly, our algorithm is not optimal when both the altimetric accuracy of the input points is poor and the point density

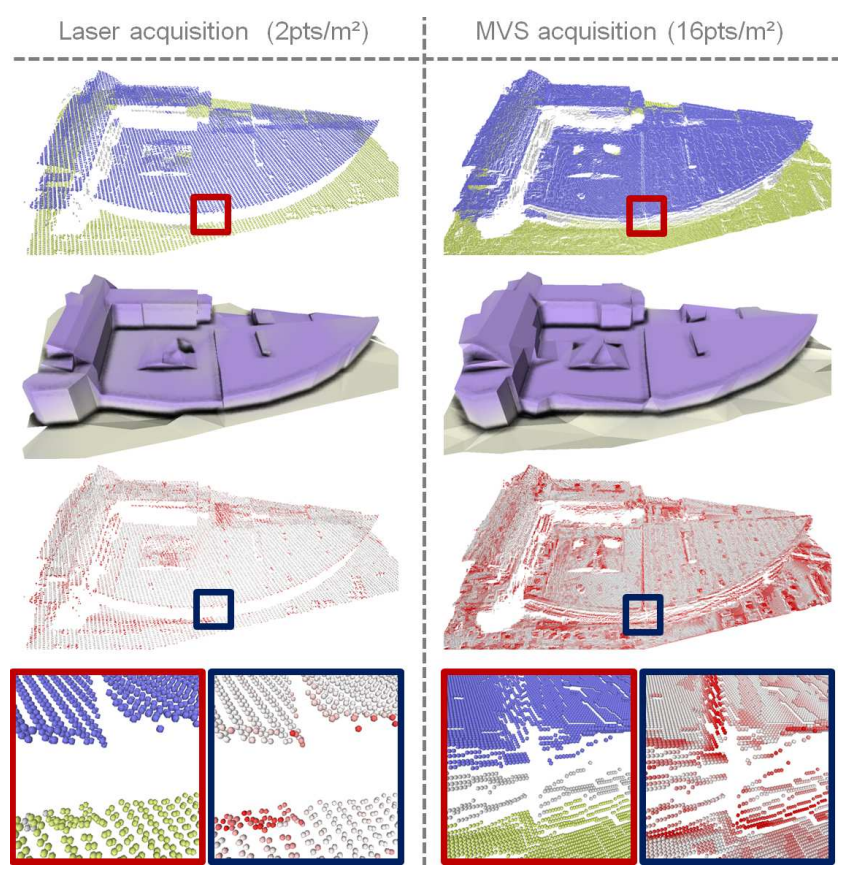

Fig. 17 Impact of the acquisition system type on our approach(from top to bottom) the classified point clouds, the obtained $3 \mathrm{D}$-models, the input clouds with the points colored according to their distance to the associated 3D-models [color code: see Fig. 11], and some associated crops. The 3D-model from MVS is less accurate in terms of structure contouring, but is of similar quality concerning the surface recovery. The laser-based 3D-model (left) has a mean error to the input data slightly lower than the MVSbased 3D-model one (right), i.e. $0.26 \mathrm{~m}$ vs $0.33 \mathrm{~m}$.

is weak, typically with low resolution Digital Surface Models, i.e. $>0.5 \mathrm{~m}$. In such cases, it is necessary to use less generic methods based on very strong urban assumptions, such as the structural-based approach of Lafarge et al. (2010a), in order to compensate for the poor quality of the data. Fourthly, the scene scale must be known in order to obtain robust results since the parameter tuning relies on the metric system. For some MVS datasets roughly geo-located, an estimation of the scene scale can thus be preliminarily required.

\section{Conclusion}

We propose an original approach for modeling large urban environments from 3D-point data. An important strength of the algorithm compared to existing methods is the complete and realistic semantized description of urban scenes by simultaneously reconstructing buildings, trees and topologically complex ground surfaces, but also the original hybrid representation of buildings combining a high level of generalization and compaction. Moreover, a general mathematical formulation for roof section arrangement problems is defined, the 

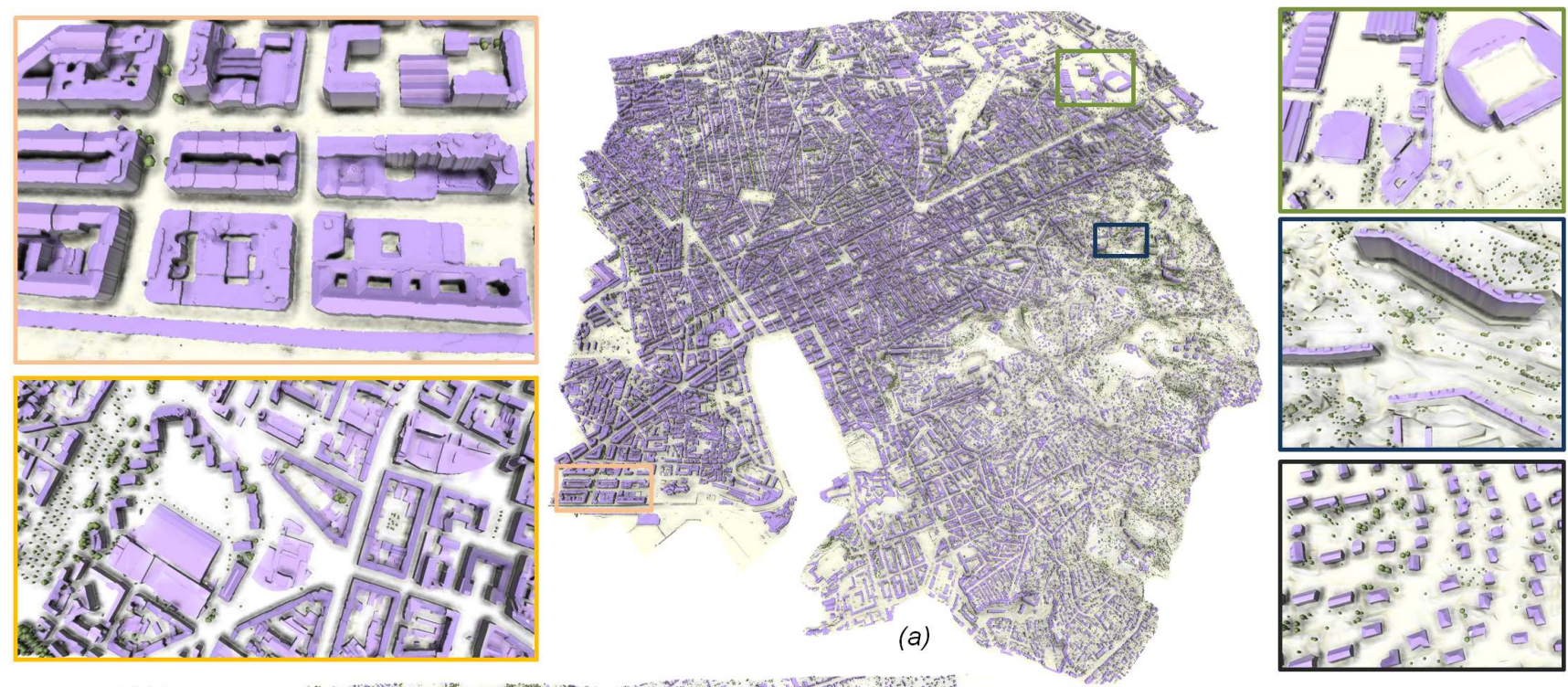

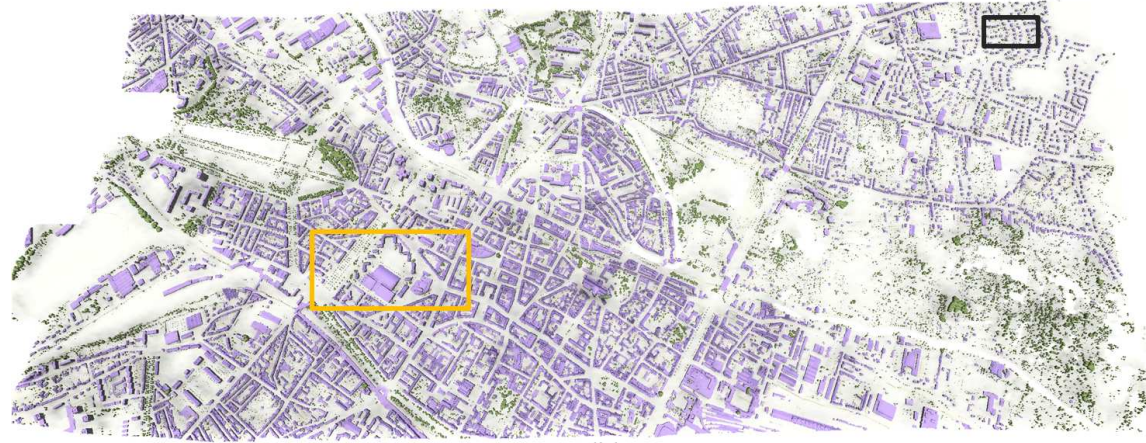

(b)

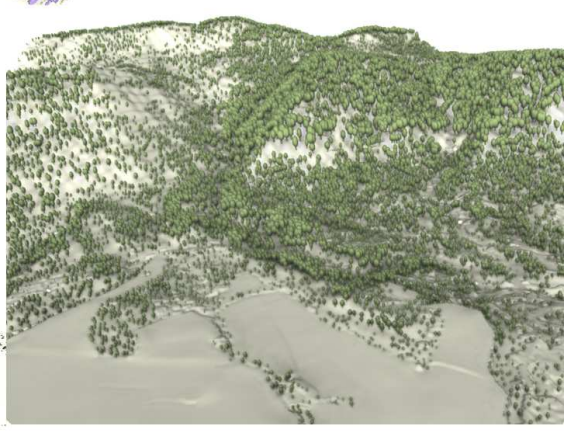

(c)

\begin{tabular}{|c|c|c|c|c|c|c|c|}
\hline & $\begin{array}{c}\text { \#input points } \\
\left(\times 10^{6}\right)\end{array}$ & $\begin{array}{c}\text { area } \\
\left(\mathrm{km}^{2}\right)\end{array}$ & $\begin{array}{c}\text { altimetric variation } \\
(\mathrm{m})\end{array}$ & $\begin{array}{c}\text { \#primitives } \\
\left(\times 10^{3}\right)\end{array}$ & $\begin{array}{c}\text { \#trees } \\
\left(\times 10^{3}\right)\end{array}$ & $\begin{array}{c}\text { computing time } \\
(\text { hour })\end{array}$ & $\begin{array}{c}\text { compaction } \\
(\mathrm{Mo})\end{array}$ \\
\hline Marseille, France (a) & 38.67 & 19.8 & 192 & 108.6 & 35.7 & 2.52 & 131 \\
\hline Amiens, France (b) & 24.52 & 11.57 & 76 & 56.7 & 22.8 & 1.34 & 93 \\
\hline Mountain area (c) & 22.67 & 3.41 & 525 & 0.01 & 21.1 & 0.31 & 34 \\
\hline
\end{tabular}

Fig. 18 Reconstruction of three large scenes with some performance statistics and crops on various types of urban landscapes.

first to date to our knowledge which works in nonrestricted contexts.

In future works, it would be interesting to improve the parallelization scheme of the energy minimization by using Graphics Processing Units (GPU). Another interesting challenge is to adapt our approach to point clouds generated from internet photo collections (Agarwal et al., 2009; Frahm et al., 2010; Furukawa et al., 2010) which contain more outliers and have spatial distributions highly heterogeneous.

\section{Acknowledgments}

We are grateful to the GDR Isis for the partial financial support, and to the reviewers for their helpful comments. We thank Qian-Yi Zhou for the two Atlanta high density Lidar scan samples (Fig. 10 and 15), Sean Belshaw from Optech for the Toronto Lidar scan sample, and the French Mapping Agency for the other datasets, including the DSM-based point clouds.

\section{References}

Agarwal, S., Snavely, N., Simon, I., Seitz, S., Szeliski, R., 2009. Building Rome in a day. In: ICCV. Kyoto, Japan.

Baillard, C., Zisserman, A., 1999. Automatic reconstruction of piecewise planar models from multiple views. In: CVPR. Los Alamitos, US.

Banno, A., Masuda, T., Oishi, T., Ikeuchi, K., 2008. Flying laser range sensor for large-scale site-modeling 
and its applications in Bayon digital archival project. IJCV 78 (2-3).

Boykov, Y., Veksler, O., Zabih, R., 2001. Fast approximate energy minimization via graph cuts. PAMI 23 (11).

Briese, C., Pfeifer, N., Dorninger, P., 2002. Applications of the robust interpolation for DTM determination. In: PCV. Graz, Austria.

Carlberg, M., Gao, P. ang Chen, G., Zakhor, A., 2009. Classifying urban landscape in aerial lidar using 3d shape analysis. In: ICIP. Cairo, Egypt.

CGAL, 2011. www.cgal.org.

Chauve, A.-L., Labatut, P., Pons, J.-P., 2010. Robust piecewise-planar $3 \mathrm{D}$ reconstruction and completion from large-scale unstructured point data. In: CVPR. San Francisco, US.

Chen, J., Chen, B., 2008. Architectural modeling from sparsely scanned range data. IJCV 78 (2-3).

Coughlan, J. M., Yuille, A. L., 2000. The Manhattan world assumption: Regularities in scene statistics which enable Bayesian inference. In: NIPS. Denver, US.

Dick, A., Torr, P., Cipolla, R., 2004. Modelling and interpretation of architecture from several images. IJCV 60(2).

Frahm et al., J.-M., 2010. Building Rome on a cloudless day. In: ECCV. Hersonissos, Greece.

Frueh, C., Zakhor, A., 2004. An automated method for large-scale, ground-based city model acquisition. IJCV 60 (1).

Furukawa, Y., Curless, B., Seitz, S., Szeliski, R., 2009. Manhattan-world stereo. In: CVPR. Miami, US.

Furukawa, Y., Curless, B., Seitz, S., Szeliski, R., 2010. Towards internet-scale multi-view stereo. In: CVPR. San Francisco, US.

Gallup, D., Frahm, J., Pollefeys, M., 2010. Piecewise planar and non-planar stereo for urban scene reconstruction. In: CVPR. San Francisco, US.

Garland, M., Heckbert, P., 1997. Surface simplification using quadric error metrics. In: SIGGRAPH. Los Angeles, US.

Golovinskiy, A., Kim, V., Funkhouser, T., 2009. Shapebased recognition of $3 \mathrm{D}$ point clouds in urban environments. In: ICCV. Kyoto, Japan.

Haala, N., Kada, M., 2010. An update on automatic 3D building reconstruction. Journal of Photogrammetry and Remote Sensing 65 (6).

Han, F., Tu, Z. W., Zhu, S. C., 2004. Range image segmentation by an effective jump-diffusion method. PAMI 26 (9).

Lafarge, F., Descombes, X., Zerubia, J., PierrotDeseilligny, M., 2010a. Structural approach for building reconstruction from a single DSM. PAMI 32 (1).
Lafarge, F., Keriven, R., Bredif, M., Vu, H., 2010b. Hybrid multi-view reconstruction by jump-diffusion. In: CVPR. San Francisco, US.

Lafarge, F., Mallet, C., 2011. Building large urban environments from unstructured point data. In: ICCV. Barcelona, Spain.

Leberl, F., Irschara, A., Pock, T., Meixner, P., Gruber, M., Scholz, S., Wiechert, A., 2010. Point clouds: Lidar versus 3d vision. Photogrammetric Engineering and Remote Sensing 76(10).

Li, S., 2001. Markov Random Field Modeling in Image Analysis. Springer.

Mallet, C., Bretar, F., 2009. Full-waveform topographic lidar: State-of-the-art. Journal of Photogrammetry and Remote Sensing 64 (1).

Marshall, D., Lukacs, G., Martin, R., 2001. Robust segmentation of primitives from range data in the presence of geometric degeneracy. PAMI 23 (3).

Matei, B., Sawhney, H., Samarasekera, S., Kim, J., Kumar, R., 2008. Building segmentation for densely built urban regions using aerial lidar data. In: CVPR. Anchorage, US.

Mayer, H., 2008. Object extraction in photogrammetric computer vision. Journal of Photogrammetry and Remote Sensing 63 (2).

Muller, P., Wonka, P., Haegler, S., Ulmer, A., Van Gool, L., 2006. Procedural modeling of buildings. In: SIGGRAPH. Boston.

Munoz, D., Bagnell, J. A., Vandapel, N., Hebert, M., 2009. Contextual classification with functional maxmargin Markov networks. In: CVPR. Miami, US.

Pollefeys et al., M., 2008. Detailed real-time urban 3D reconstruction from video. IJCV 78 (2-3).

Poullis, C., You, S., 2009. Automatic reconstruction of cities from remote sensor data. In: CVPR. Miami, US.

Schnabel, R., Wahl, R., Klein, R., 2007. Efficient RANSAC for point-cloud shape detection. Computer Graphics Forum 26 (2).

Sinha, S. N., Steedly, D., Szeliski, R., 2009. Piecewise planar stereo for image-based rendering. In: ICCV. Kyoto, Japan.

Strecha, C., Von Hansen, W., Van Gool, L., Fua, P., Thoennessen, U., 2008. On benchmarking camera calibration and multi-view stereo for high resolution imagery. In: CVPR. Anchorage, US.

Toshev, A., Mordohai, P., Taskar, B., 2010. Detecting and parsing architecture at city scale from range data. In: CVPR. San Francisco, US.

Tse, R., Gold, C., Kidner, D., 2007. Using the delaunay triangulation/ voronoi diagram to extract building information from raw lidar data. In: Proc. of International Symposium on Voronoi Diagrams in Science 
and Engineering. Urmchi, China.

Vanegas, C., Aliaga, D., Benes, B., 2010. Building reconstruction using Manhattan-world grammars. In: CVPR. San Francisco, US.

Vosselman, G., Kessels, P., Gorte, B., 2005. The utilisation of airborne laser scanning for mapping. Int. Jour. of Applied Earth Observation and Geoinformation 6 (3-4).

Vu, H., Keriven, R., Labatut, P., Pons, J., 2009. Towards high-resolution large-scale multiview. In: CVPR. Miami, US.

Weiss, Y., Freeman, W., 2001. On the optimality of solutions of the max-product belief propagation algorithm in arbitrary graphs. IEEE Trans. on Information Theory 47 (2).

Xu, G., Zhang, Z., 1996. Epipolar geometry in stereo, motion and object recognition. Kluwer.

$\mathrm{Xu}$, H., Gossett, N., Chen, B., 2007. Knowledge and heuristic-based modeling of laser-scanned trees. Trans. on Graphics 26 (4).

Zebedin, L., Bauer, J., Karner, K., Bischof, H., 2008. Fusion of feature- and area-based information for urban buildings modeling from aerial imagery. In: ECCV. Marseille, France.

Zhou, Q., Neumann, U., 2010. 2.5d dual contouring: A robust approach to creating building models from aerial lidar point clouds. In: ECCV. Hersonissos, Greece.

Zhu, Z., Kanade, T., 2008. Special issue on modeling and representations of large-scale 3D scenes. IJCV $78(2-3)$. 